\title{
Unexpected complexity in the interference activity of a cloned influenza defective interfering RNA
}

\author{
Bo Meng ${ }^{1}$, Kirsten Bentley ${ }^{2}$, Anthony C. Marriott ${ }^{3}$, Paul D. Scott ${ }^{4}$, Nigel J. Dimmock ${ }^{5}$ and Andrew J. Easton ${ }^{*}$
}

\begin{abstract}
Background: Defective interfering (DI) viruses are natural antivirals made by nearly all viruses. They have a highly deleted genome (thus being non-infectious) and interfere with the replication of genetically related infectious viruses. We have produced the first potential therapeutic DI virus for the clinic by cloning an influenza A DI RNA (1/244) which was derived naturally from genome segment 1 . This is highly effective in vivo, and has unexpectedly broad-spectrum activity with two different modes of action: inhibiting influenza A viruses through RNA interference, and all other (interferon-sensitive) respiratory viruses through stimulating interferon type I.

Results: We have investigated the RNA inhibitory mechanism(s) of DI 1/244 RNA. Ablation of initiation codons does not diminish interference showing that no protein product is required for protection. Further analysis indicated that 1/244 DI RNA interferes by replacing the cognate full-length segment 1 RNA in progeny virions, while interfering with the expression of genome segment 1, its cognate RNA, and genome RNAs 2 and 3, but not genome RNA 6, a representative of the non-polymerase genes.
\end{abstract}

Conclusions: Our data contradict the dogma that a DI RNA only interferes with expression from its cognate full-length segment. There is reciprocity as cloned segment 2 and 3 DI RNAs inhibited expression of RNAs from a segment 1 target. These data demonstrate an unexpected complexity in the mechanism of interference by this cloned therapeutic DI RNA.

Keywords: Influenza virus, Defective interfering RNA, Replication, Interference

\section{Background}

Viral replication frequently generates progeny genomes that contain extensive deletions [1-4]. This is thought to be a consequence of the polymerase detaching from the initial template and reattaching elsewhere on the same or different template. The truncated genomes are unable to generate infectious virus particles and are thus functionally defective. During a single infectious cycle a large number of different defective genomes can be generated, each lacking a different portion of the genome. Molecules containing the necessary signals will be replicated and packaged into virus particles. Some, but not all, of these defective genomes are also capable of interfering with the growth of the parental virus from which they were derived and are known as defective-interfering (DI)

\footnotetext{
* Correspondence: a.jeaston@warwick.ac.uk

${ }^{5}$ School of Life Sciences, University of Warwick, Coventry CV4 7AL, UK Full list of author information is available at the end of the article
}

genomes [5]. The ability to interfere with virus multiplication suggests that these DI genomes could act as antiviral agents in the clinic [6,7].

The influenza A virus genome comprises 8 segments of single-stranded negative-sense RNA (vRNA) in the form of ribonucleoprotein (RNP) complexes, and one copy of each of these segments is required to make an infectious virus particle [8-10]. Influenza virus infections can generate DI RNAs, which can interfere with virus replication. No copyback or snapback influenza DI RNAs have been detected, and most influenza DI RNAs have a major (approximately 80\%) internal deletion, while retaining the cis-acting signals required for replication and packaging into virus particles [11-13]. DI RNA is incorporated into a particle but the resulting DI virus cannot replicate autonomously since it is unable to synthesise the protein(s) normally encoded by the deleted segment. Replication of a DI virus thus requires complementation by infectious virus. However, DI RNAs may 
be able to express truncated proteins which could play a role in interference of infectious virus replication $[14,15]$.

Influenza virus genome replication commences with synthesis of positive-sense (cRNA) copies of the vRNAs of the infecting virus, and in turn these are used as templates for synthesis of new vRNAs [10]. vRNA is also used as the template for mRNA transcription. Unlike cRNA synthesis, mRNA synthesis is initiated using a primer cleaved from the capped $5^{\prime}$ end of host mRNAs and its synthesis terminates before the end of the template vRNA, prior to polyadenylation [16-19]. Thus the mRNA differs from cRNA in having the primer-derived 5 '-extension, being truncated, and being polyadenylated at the $3^{\prime}$ end. The non-coding termini of each segment are crucial for RNA synthesis, and contain a conserved, approximately 12 nucleotide (nt), sequence at the $5^{\prime}$ end which is almost exactly complemented at the $3^{\prime}$ end [10].

Synthesis of influenza virus RNAs is carried out by a virus-encoded RNA-dependent RNA polymerase present within a RNP complex consisting of the vRNA or cRNA in association with the virus NP protein and the heterotrimeric virus RNA polymerase comprised of the PB1, PB2 and PA proteins. The latter are encoded by vRNA segments 2, 1 and 3, respectively. Mutations in PB2 and PA can decouple transcription (mRNA synthesis) from replication (vRNA and cRNA synthesis), such that a mutant polymerase is defective in replication but not in transcription or vice versa $[20,21]$. Although initiation of mRNA and cRNA synthesis differ in mechanism, a 'switch' directing the polymerase to synthesise one or the other has not been clearly demonstrated, and both can be synthesised from vRNP in a cell-free system [22, 23]. The polymerase complex binds to conserved sequences at the $5^{\prime}$ and $3^{\prime}$ ends of vRNA and cRNA [24-26]. The termini of cRNA differ from those of vRNA at several nucleotide positions, and critical residues of each may interact with distinct regions of the PB1 protein [27, 28]. Synthesis of vRNA and cRNA can also be affected differentially by mutations in the NP or NS1 proteins [29-31], or by the presence or absence of the NS2 protein [32].

Little progress has been made towards understanding the mechanism of interference by DI viruses. For snapback/ copyback vesicular stomatitis virus (VSV) DI RNAs that have a high degree of double-strandedness, but not VSV DI RNAs generated by central deletion, the key factor in interference seems to be the extent of terminal complementarity of the DI RNA such that a longer DI RNA with more extensive terminal complementarity out-competes a shorter DI RNA of the same structure [33-35]. In addition, lack of a transcriptional promoter in snapback/copyback DI RNAs gives them a replicative advantage over full-length genomic RNA or centrally deleted DI RNAs that can carryout transcription [3, 13, 36-39]. These properties may explain the observed preponderance of rhabdovirus copyback/snapback DI RNAs.
For DI RNAs generated by a central deletion, as seen in influenza viruses, it has been suggested that interference with RNA synthesis involves competition between the DI RNA and genomic RNA for limiting viral or host factor(s), and/or the much shorter DI RNA may have a more rapid rate of synthesis than its cognate genomic RNA giving it a competitive advantage, although there is little experimental evidence supporting this $[3,13,36-40]$.

Most studies of DI influenza virus-mediated interference to date have been carried out with naturally occurring preparations, and are compromised by the presence of mixtures of several different defective RNA sequences $[4,41]$. This problem was solved using reverse genetics to generate virus stocks containing a molecularly defined naturally occurring DI RNA. Called $1 / 244$ to denote its derivation from segment 1 and the number of nucleotides retained at the $3^{\prime}$ end of the positive-sense RNA, it is propagated and efficiently maintained by infectious virus often referred to as 'helper' virus. When delivered intranasally as an influenza virus particle, 1/244 DI virus confers complete protection in mice from a lethal challenge with several different subtypes of influenza A virus (homologous protection) and is more effective than oseltamivir in ferrets in combatting pandemic influenza A/ California/04/09 [42]. However, the molecular basis of this homologous protection by $1 / 244$ DI virus is not known. In addition, $1 / 244$ DI virus also protects from the genetically heterologous influenza $\mathrm{B}$ virus and a murine pneumovirus in a dose-dependent manner through its ability to induce the local expression of interferon type I, although induction of interferon is not required for protection against influenza A virus [43, 44]. Here we show that interference with influenza A virus mediated by molecularly cloned $1 / 244$ DI RNA does not require expression of a protein product from the deleted segment 1 but results from both competition with the packaging of full-length cognate virion segment 1 RNA, and by inhibiting the synthesis and/or accumulation of RNA directed by segment 1 RNA. Unexpectedly $1 / 244$ DI RNA also interfered with the expression of RNAs from full-length segments 2 and 3, which encode the other polymerase proteins, but not from full-length segment 6 .

\section{Methods \\ Plasmids}

The plasmids encoding the 8 gene segments of the $\mathrm{A} /$ WSN strain of A/WS/33 (H1N1) and plasmids expressing the polymerase proteins and NP [45], and the construct expressing 1/244 DI RNA (1/244), derived from a naturally occurring DI virus, were as previously described [46]. 1/244 RNA comprises $395 \mathrm{nt}$ with $151 \mathrm{nt}$ from the $5^{\prime}$ end and 244 nt from the $3^{\prime}$ end of the positive-sense cognate RNA and was derived naturally from segment 1 
of A/Puerto Rico/8/34 (H1N1) (Fig. 1). The segment 1 target, segment 1-GFP, expressing the green fluorescent protein (GFP), was created by amplifying the GFP open reading frame by PCR from pEGFP-N1 (Clontech) using primers 5'ATGGTCTCTACTGATGGTGAGCAAGGGCGAG and 5'ATGAAGACAATCTCTTACTTG TACAGCTCGTCCA. The product was inserted between the BpiI and Eco31I sites of pPolI-220 [47] such that the GFP ORF is in-frame with the PB2 ORF, giving plasmid seg 1-GFP which expresses segment 1-GFP RNA (Fig. 1). The GFP reporter retains the exact 5' (220 nt) and 3' (48 nt) terminus of segment 1 and is cognate for $1 / 244$ DI RNA. A naturally occurring segment 2 DI (2/265; comprising $452 \mathrm{nt}$ in total with $265 \mathrm{nt}$ from the $3^{\prime}$ end and 187 nt from the $5^{\prime}$ end of the positive-sense cognate RNA) was isolated from a DI A/equine/Newmarket/7339/ 79 (H3N8) virus preparation (Fig. 1) [48] by RT-PCR amplification, and subsequently cloned into a pPolI-SapIT expression vector [49]. A further naturally occurring segment 3 DI RNA (3/262; comprising 469 nt in total with 262 nt from the $3^{\prime}$ end and 207 nt from $5^{\prime}$ end of the positive-sense cognate RNA) was isolated from a DI A/ WSN preparation, and was amplified and cloned as above (Fig. 1). The DI RNAs encoded by the various plasmids retain the exact nucleotide sequences from the termini of the genome segments of the viruses from which they were derived and do not contain any mutations in positions known to have an effect on replication or packaging. Two sequential steps of site-directed mutagenesis were carried out to mutate the start codons in the 1/244 DI RNA. A pair of primers (GTTCTTTTATTCTTTCgATATTGAAT ATAATTG and CAATTATATTCAATATcGAAAGAAT AAAAGAAC) was used for site-directed mutagenesis to convert the first AUG to AUC using a pPolI-244 plasmid as template and Pfu DNA polymerase (Promega). The lower case letters indicate the position of the mutations.

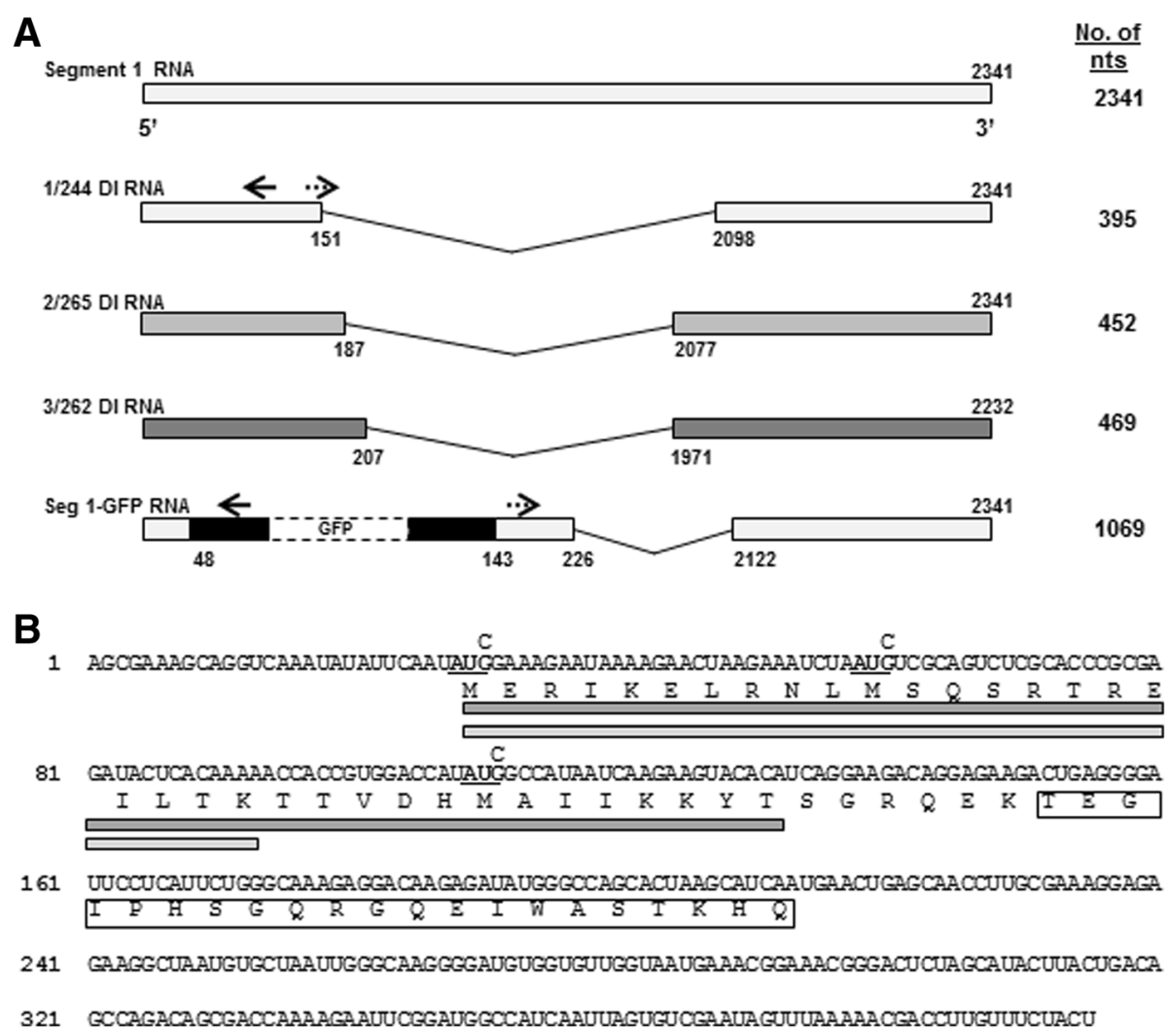

Fig. 1 a. Schematic diagram of influenza segment 1244 DI RNA (1/244), segment 2265 DI RNA (2/265), segment 3262 DI RNA (3/262), and the Seg 1-GFP RNA expressed from plasmids. The names derive from the 244,265 and $262 \mathrm{nt}$ remaining at the $3^{\prime}$ end of the cognate vRNAs respectively.The total number of nucleotides comprising each RNA is shown on the right. The nucleotide positions of the breakpoints that result in the deleted genome RNAs used in this study (positive-sense, $5^{\prime}$ to $3^{\prime}$ ) are indicated. Solid arrows indicate the primers used in the primer extension assays for cRNA and mRNA analyses and dashed arrows indicate primers used for vRNA analyses. For the construction of Seg 1-GFP RNA see Methods. b. Sequence of 1/244 DI RNA in cRNA sense indicating the open reading frame, and the predicted protein sequence in single letter amino acid code. The 35 residue PB1 binding domain of PB2 is indicated by the dark grey box, and the 22 residue mitochondrial interaction domain of PB2 is indicated by the light grey box. The boxed amino acid sequence downstream from the central deletion that gave rise to 1/244 DI RNA do not arise from the PB2 ORF. The three $G \rightarrow C$ mutations at nucleotide positions 30,60 and 111 used to mutate the in-frame AUG initiation codons are shown in bold and underlined 
The second round of site-directed mutagenesis used primers that altered the second and third start codons to AUC using the construct produced from the first round of mutagenesis (CTTCTTGATTATGGCcATATGGTCCAC GGTGGTTTTTGTGAGTATCTCGCGGGTGCGAGAC TGCGAcATTAGATTTCTTAGT and ACTAAGAAA TCTAATgTCGCAGTCTCGCACCCGCGAGATACTC ACAAAAACCACCGTGGACCATATgGCCATAATCA AGAAG). All constructs were confirmed by sequencing.

\section{Transfection and recovery of infectious virus}

Human 293T cells (American Type Culture Collection) were transfected as previously described. Briefly, for northern blot analysis, a well of $70 \%$ confluent $293 \mathrm{~T}$ cells in a 12-well plate was transfected using TransIT LT1 transfection reagent (Mirus) with 8 Poll expression plasmids encoding virion sense RNA, and cDNA plasmids for expression of PB2, PB1, PA and NP proteins, with or without pPolI-244. The transfected cells were then incubated at $37{ }^{\circ} \mathrm{C}$ overnight before co-culture with MDCK cells (obtained from the American Type Culture Collection) in a $25 \mathrm{~cm}^{2}$ flask. Total cellular RNA was extracted with $2 \mathrm{ml}$ Trizol reagent per sample (Invitrogen) on days 1, 2 and 3 after co-culture while tissue culture fluid was collected for virus titration and RNA extraction. Virions were purified by ultracentrifugation. RNA was extracted with phenol/chloroform, and then ethanol precipitated.

For preparation of virus stocks containing DI 1/244 and $1 / 244$ KO DI RNA, virus in tissue culture fluids produced by transfection was passaged once in embryonated chicken's eggs and allantoic fluids were harvested to produce a stock of virus. The virus produced in embryonated chicken's eggs is a mixture of $1 / 244$ DI virus or $1 / 244$ AUG KO DI virus packaged in A/WSN virion proteins and infectious helper A/WSN strain of A/WS/ 33 (H1N1) virus. These were purified by differential centrifugation through sucrose, and resuspended in PBS. Stocks were standardized according to their haemagglutination titre and stored in liquid nitrogen. The DI virus stock was UV-irradiated to remove helper virus infectivity using a short burst (40 s) of UV irradiation at $253.7 \mathrm{~nm}\left(0.64 \mathrm{~mW} / \mathrm{cm}^{2}\right)$. This is 'active DI virus'. Longer UV irradiation $(8 \mathrm{~min})$ inactivates the protective activity in mice, but does not affect haemagglutinin or neuraminidase activities, and so controls for any immune system-stimulating or receptor-blocking effects of 1/244 DI virus particles ('inactivated DI virus').

\section{Infectivity assay}

MDCK cell monolayers in 96-well plates were infected with supernatant containing rescued A/WSN as described previously [44]. After $1 \mathrm{~h}$ for attachment of virus, the monolayer was washed with $\mathrm{PBS}$, and incubated in maintenance medium overnight at $33{ }^{\circ} \mathrm{C}$. Cells were then fixed with $4 \%(v / v)$ formaldehyde, washed and blocked with $5 \%(w / v)$ milk powder in PBS. The infected cells were probed with a monoclonal antibody specific for the HA of A/WSN in PBS containing 0.1\% Tween 20. After washing, goat anti-mouse IgG-alkaline phosphatase conjugate (Sigma) in TBS containing $0.1 \%$ Tween 20 was added, and infected cells were detected with nitrotetrazolium blue chloride/BCIP (Sigma) in Tris-buffered magnesium chloride and sodium chloride $(0.1 \mathrm{M}, \mathrm{pH} 9.5)$. The infectivity titre was determined by counting at least 50 positively stained cells (foci) at an appropriate dilution in each of the triplicate wells. The mean number of counts was determined to give a titre in focus-forming units (f.f.u.) $\mathrm{ml}^{-1}$.

\section{Protection of mice from influenza with DI virus}

To assess the degree of in vivo protection afforded by DI virus, groups of $5 \mathrm{C} 3 \mathrm{H} / \mathrm{He}-\mathrm{mg}$ mice were each inoculated intranasally under light ether anaesthesia with A/WSN alone (10 $\mathrm{LD}_{50}$ or 1000 f.f.u.), active DI virus alone, a mixture of A/WSN + active DI virus, or A/WSN + inactivated DI virus. The amounts of DI $1 / 244$ used was the same as used in previous studies of protection from disease and mice were subsequently monitored for clinical disease according to our standard protocol [43, 44]. The severity of disease was scored using the standard scoring system with a score of 1 indicating a healthy animal and a score of 5 indicating death with a range of severity of cinical signs for intervening scores as described previously [43, 44]. Animals were assessed daily and data are expressed as the mean score for each group. Surviving mice were challenged 3 weeks after infection with a high dose of A/WSN $\left(10,000 \mathrm{LD}_{50}\right)$ to determine their immune status. All work with animals was governed by a licence approved by the UK Home Office and complied with national regulations. Animal work was approved by the University of Warwick Animal Welfare and Ethical Review Board as required by the Animals (Scientific Procedures) Act (1986) governing animal experimentation in the UK. Animals culled for humane reasons were subjected to an approved procedure to minimise suffering.

\section{RNA extraction and primer extension}

For primer extensions, each well of a 6 well plate containing $70 \%$ confluent $293 \mathrm{~T}$ cells was transfected with $1 \mu \mathrm{g}$ each of the PB2, PB1, PA and NP cDNA expression plasmids plus various amounts of either a DI plasmid, pPolI-PB2, or pPolI-NA together with $1 \mu \mathrm{g}$ of target plasmid. Cells were incubated at $37{ }^{\circ} \mathrm{C}$ and total cellular RNA was extracted from cells with Trizol at $48 \mathrm{~h}$ posttransfection.

Primer extension analysis was carried out as previously described [50], using primer mixes in order to detect vRNA, cRNA, mRNA and 5S rRNA simultaneously from 
individual samples. Total RNA $(2 \mu \mathrm{g})$ was mixed with $\left[{ }^{32} \mathrm{P}\right] 5^{\prime}$-end labelled primers and dNTP in a total volume of $13 \mu \mathrm{l}$. The mixture was heated at $65{ }^{\circ} \mathrm{C}$ for $5 \mathrm{~min}$ and placed on ice for $1 \mathrm{~min}$. 2X first Strand Buffer, $20 \mathrm{mM}$ DTT, and $100 \mathrm{U}$ SuperScript III reverse transcriptase (Invitrogen) were added and further incubated at $55^{\circ} \mathrm{C}$ for $1 \mathrm{~h}$. The reaction was terminated by heating at $95^{\circ} \mathrm{C}$ for $5 \mathrm{~min}$ with gel loading dye II (Ambion). The transcription products were resolved on a $6 \%(w / v)$ polyacrylamide gel containing $7 \mathrm{M}$ urea in TBE buffer and detected by phosphor imaging. The primers used, and the expected product sizes, are shown in Table 1 and the positions of these on the various target RNAs are demonstrated in Fig. 1a.

\section{Northern blotting}

Ten $\mu \mathrm{g}$ of total cellular RNA or $50 \%$ of the yield of purified virion RNA from each sample was analysed by glyoxal-agarose gel electrophoresis. Poly A-containing mRNA was selected using a GenElute Direct mRNA preparation kit (Sigma) according to the manufacturer's instructions. Non-polyadenylated RNA that did not bind to the column during mRNA preparation, and therefore contained the viral cRNA, was retained for further study. The RNA was transferred onto Hybond-N membrane (GE Healthcare) overnight using $20 \times$ SSC. The membrane was then baked at $80{ }^{\circ} \mathrm{C}$ for $2 \mathrm{~h}$ and hybridized with digoxigenin (DIG)-labelled probes overnight. The full-length positive-sense DIG-labelled segment 1 , segment 2 and segment 7 probes were transcribed in vitro in the presence of DIG-UTP (Roche) from PCR products containing a T7 promoter. The Roche system with a digoxigenin-specific FAb antibody fragment conjugated to alkaline phosphatase and the chemiluminescent CSPD substrate was used for detection. Blots were exposed to Fuji X-ray film until the desired density was achieved and bands were quantified by densitometry using Image (NIH).

\section{Quantitation of GFP-expressing cells}

Human 293T cells were transfected with the segment 1GFP RNA expressing plasmid, plasmids expressing PB1, $\mathrm{PB} 2, \mathrm{PA}$ and NP proteins, and increasing amounts of an additional PolI plasmid expressing a DI RNA (1/244, 2/265 and 3/262) or a full-length RNA (segment 1,4 or 6 ). At 2 days post-transfection, the cultures were examined for GFP expression. Each dataset was replicated several times and all data were included in the calculations. Digital images of the cell monolayers were taken by phase-contrast and epifluorescence microscopy. Five field fluorescence images were randomly selected and analysed for the proportion of the visualised area expressing GFP using the HCImage software (Hamamatsu). The visualisation detects cells expressing a range of GFP levels to include those that may have been transfected with different levels of the reporter plasmids. A mean was calculated to give the percentage of the GFP positive area per monolayer.

\section{Results \\ 1/244 DI RNA directs synthesis of a small mRNA in the presence of infectious helper virus}

It has been shown that a truncated form of the flu A PB2 protein is capable of inducing type I interferon and an antiviral state [15]. DI 1/244 RNA contains the $3^{\prime}$ terminal $244 \mathrm{nt}$ of the segment 1 vRNA that may direct mRNA synthesis and would consequently have the potential to encode a truncated protein containing the amino terminus of PB2 (Fig. 1a, b). We therefore investigated if the synthesis of the potential truncated protein from 1/244 DI RNA had a role in interference or protection against influenza in vivo. First, we determined if 1/244 DI

Table 1 Sequences of the primers used in this study. The primers containing mixed nucleotides were designed for detection of both A/PR8 and A/WSN-derived RNAs. Influenza virus mRNAs utilise primers that are 10-13 nt in length and the predicted sizes of the mRNA fragments reflect this heterogeneity

\begin{tabular}{|c|c|c|c|}
\hline Target RNA & Primer specificity & Primer sequence & Expected product Size/s (nt) \\
\hline \multirow[t]{2}{*}{ Seg 1-GFP } & $\mathrm{c} / \mathrm{mRNA}$ & GGACACGCTGAACTTGTGG & $142 / 152-5$ \\
\hline & VRNA & AGATAAGAGGATAATGGAAATG & 242 \\
\hline \multirow[t]{2}{*}{ DI 1/244 } & $\mathrm{c} / \mathrm{mRNA}$ & ATATGGTCCACKGTGGTITITG & $110 / 120-3$ \\
\hline & VRNA & GGAGAAGACTGAGGGGATTC & 252 \\
\hline \multirow[t]{2}{*}{ Segment 2 (PB1) } & $\mathrm{c} / \mathrm{mRNA}^{*}$ & TCCATGGTGTATCCTGTTCC & $146 / 156-9$ \\
\hline & VRNA* & TGATTTCGAATCTGGAAGGA & 134 \\
\hline \multirow[t]{2}{*}{ Segment 3 (PA) } & $\mathrm{c} / \mathrm{mRNA}^{*}$ & TGAGTGCATATTGCTGCAAAT & $146 / 156-9$ \\
\hline & VRNA* & TTCTTATCGTTCAGGCTCTT & 213 \\
\hline \multirow[t]{2}{*}{ Segment 6 (NA) } & $\mathrm{c} / \mathrm{mRNA}^{*}$ & TCCAGTATGGTTITGAYTTCCR & $160 / 170-3$ \\
\hline & VRNA* & TGGACTAGTGSGAGCATSAT & 130 \\
\hline $5 S$ rRNA* & & TCCCAGGCGGTCTCCCATCC & 100 \\
\hline
\end{tabular}

* indicates primers taken from [50] 
RNA could direct the synthesis of mRNA by analysing the viral RNAs present in cells infected with $1 / 244$ DI virus and infectious helper virus. As with all DI viruses 1/244 DI virus is replication incompetent, in this case due to the lack of a functional genome segment 1 . To study the viral RNAs synthesised from $1 / 244$ we therefore co-infected cells with $1 / 244$ virus and infectious A/WSN (H1/N1) as helper virus to provide the segment 1 encoded $\mathrm{PB} 2$ protein needed to generate 1/244 RNAs. Both viruses were generated entirely from molecular clones by reverse genetics as described previously [51] to avoid the presence of other DI RNAs. Northern blot analysis using a segment 1 specific probe detected two polyadenylated virus mRNAs, of approximately 2.3 and $0.5 \mathrm{~kb}$, in infected cells (Fig. 2a). These sizes are consistent with mRNAs predicted to be derived from the full-length genome segment 1 provided by the helper virus and by $1 / 244$ DI RNA, respectively. The positive-sense RNA seen in the non-polyadenylated RNA fraction is cRNA and, as expected, was slightly smaller than the $1 / 244$ DI-derived mRNA. These data indicate that the 1/244 DI
RNA is replicated by the infectious helper virus and acts as a template for both replication and transcription from a fully functional RNP complex equivalent to those found with the genomic RNA segments [23].

\section{Expression of a putative protein by $1 / 244$ DI RNA is not required for interference in vitro or protection against disease in vivo}

The mRNA transcribed from 1/244 DI RNA contains the translation start codon of the $\mathrm{PB} 2$ open reading frame 1 (ORF-1). This has the potential to encode a protein comprising the first 41 amino acid residues of PB2 fused to 21 amino acid residues translated from a different reading frame generated as a result of the deletion, making a protein of 62 residues in total (Fig. 1b). This putative protein contains the entire PB1-binding domain of residues 1-35 [33] and the mitochondrial localisation domain of residues 1-22 [52]. We investigated whether the putative protein was required for the previously reported protection from infection by $1 / 244$ DI RNA by
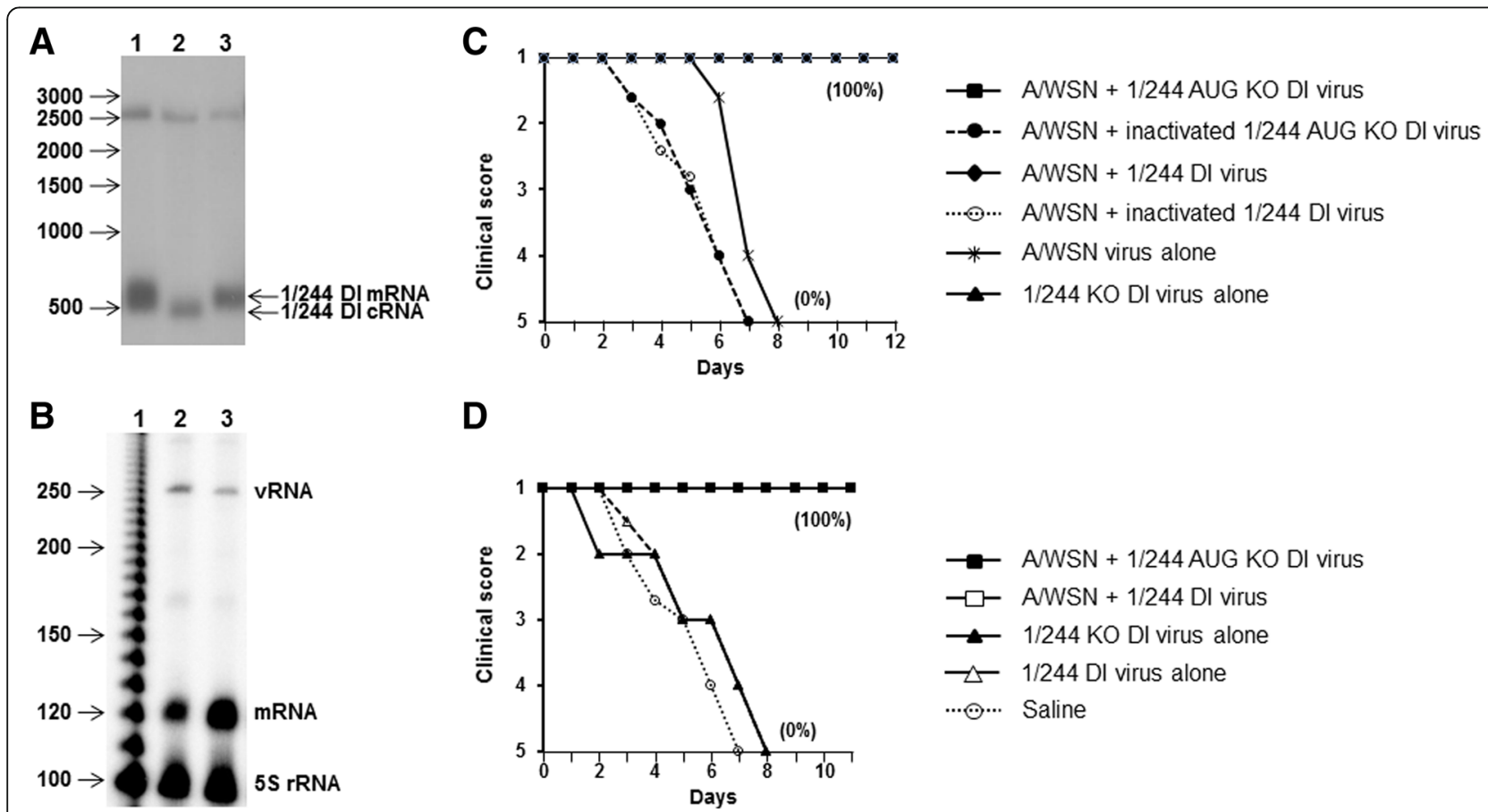

D
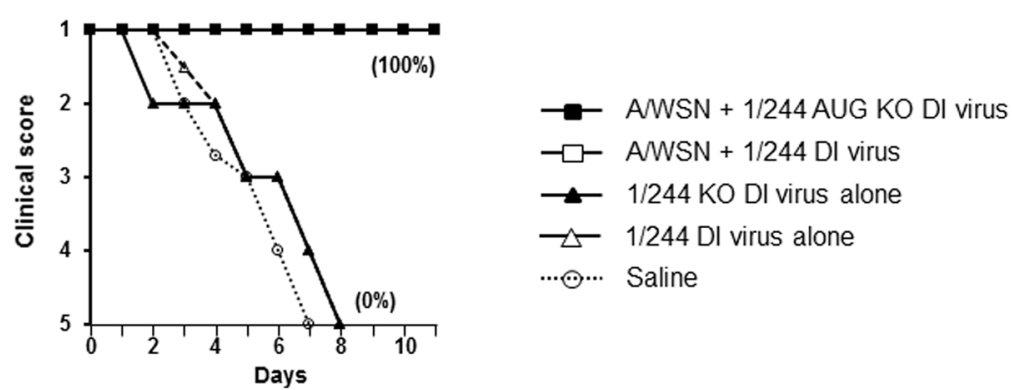

Fig. 2 Detection of RNAs synthesised by 1/244 DI RNA and 1/244 KO DI RNA by Northern blot and primer extension and protection of mice from influenza. a. Northern blot of RNA extracted from cells $48 \mathrm{~h}$ after infection with 1/244 DI virus and helper virus to detect positive-sense influenza RNA transcribed from genome segment 1 and the 1/244 RNA itself. Lane 1: total RNA; lane 2: non-polyadenylated RNA; lane 3 polyadenylated mRNA. The positions of size markers (nt) are indicated. b. Viral RNAs synthesised by 1/244 DI RNA and 1/244 AUG KO DI RNA and detected by primer extension. Samples were taken $48 \mathrm{~h}$ after transfection with plasmids expressing Seg 1-GFP, PB1, PB2, PA and NP proteins and 1/244 and 1/244 AUG KO DI RNAs. Lane 1: a 10 nt size ladder; lane 2: RNA made in the presence of 1/244 DI RNA; lane 3: RNA made in the presence of 1/244 AUG KO DI RNA. The positions of VRNA and mRNA are indicated. 5 S ribosomal RNA was measured as a loading control. c. Mice were inoculated intranasally with AWSN alone (10 LD 50,1000 f.f.u.), A/WSN + 1/244 AUG KO DI virus, A/WSN + 1/244 DI virus, A/WSN + inactivated 1/244 AUG KO DI virus, A/WSN + inactivated 1/244 DI virus, or saline alone. Each DI virus comprised $1 \mu \mathrm{g}$ protein. d. Surviving mice were challenged with a high dose of ANSN $\left(10,000 \mathrm{LD}_{50}\right)$ at 3 weeks post-infection to determine their immune status. Panels $C$ and $D$ show the mean clinical score. In panel C ANSN + 1/244 DI, 1/244 KO DI only, 1/244 DI only, and mock are all hidden under A/WSN + 1/244 AUG KO DI with a clinical score of 1 . The percentage of surviving mice is shown in parenthesis 
mutating the sequence to remove the translation initiation codons. The PB2 ORF has three possible AUG start codons and to be sure there could be no translation initiation all three possible start codons were mutated (to AUC). The new RNA is referred to as 1/244 AUG KO DI RNA.

RNAs were harvested $48 \mathrm{~h}$ after transfection of 293T cells with plasmid encoding either the $1 / 244$ DI RNA or the 1/244 AUG KO DI RNA together with plasmids expressing the PB2, PB1, PA and NP proteins. Primer extension analysis, using a mixture of primers to detect both positive- and negative-sense RNAs simultaneously (Table 1), showed that $1 / 244$ and $1 / 244$ AUG KO synthesised both types of RNA with the predicted sizes, confirming that both genomes acted as templates for RNA synthesis in transfected cells. Densitometric analysis followed by normalisation against the 5S rRNA loading control showed that the levels of mRNA synthesized by $1 / 244$ and $1 / 244$ AUG KO DI RNAs were similar (mRNA/5S ratios of 0.87 and 0.9 , respectively) confirming that transcription was unaffected by the mutations in the 1/244 AUG KO DI RNA (Fig. 2b).

In our experience all stocks of influenza virus contain some level of a complex population of DI viruses, which makes examination of the activity of any individual transfected DI RNA in a cell culture infection model technically impossible. In such experiments it is necessary to use a high level of infectious virus which results in a prominent signal from the naturally occurring DI RNAs that obscures the signal from the transfected plasmid expressing 1/244 DI RNA (unpublished data). We have therefore used an established in vitro RNP reconstitution approach [53-55] to examine the ability of DI RNAs to interfere with the expression of green fluorescent protein (GFP) from a Seg 1-GFP construct. Here the coding region of GFP is inserted in-frame into a deleted influenza segment 1 RNA (Fig. 1a). This system also distinguishes effects of DI RNA on RNA synthesis from any effect on RNA packaging as plasmids encoding key structural proteins (HA, NA, M1 and M2) are not included [56]. The interfering ability of $1 / 244$ and $1 / 244$ AUG KO RNAs were determined by transfecting $293 \mathrm{~T}$ cells with the relevant plasmids and plasmids expressing Seg 1-GFP and PB1, PB2, PA and NP proteins. 1/244 and 1/244 AUG KO RNAs both strongly inhibited fluorescence in a dose-dependent manner (Fig. 3a) with over $90 \%$ inhibition at the dose of $0.5 \mu \mathrm{g}$ for both $1 / 244$ or $1 /$ 244. AUG KO plasmids (Fig. 3b).

To determine whether the in vitro interference is also reflected in an infection model, we compared the abilities of $1 / 244$ DI and 1/244 AUG KO DI RNA to protect mice from disease that normally follows challenge with influenza A/WSN (H1N1). Virus stocks containing either 1/244 DI or 1/244 AUG KO DI RNA were produced using reverse genetics as before [51] and mice were infected intranasally with either A/WSN alone, A/WSN and 1/244 DI virus, or A/WSN and 1/244 AUG KO DI virus. Additional infected groups received DI virus which had been UV-irradiated for 8 min to destroy DI protecting activity and to control for any non-specific effects of the DI virus inoculum [43, 44]. Mice were monitored for clinical disease. Figure 2c shows that the A/WSN virus-infected control mice all became seriously ill, and had to be culled. In contrast, none of the infected mice also receiving 1/244 DI virus or $1 / 244$ AUG KO DI virus developed any sign of clinical disease. All mice treated with $\mathrm{A} / \mathrm{WSN}+1 / 244$ DI virus or $1 / 244 \mathrm{KO} \mathrm{DI}$ virus alone were also healthy throughout and these data points are obscured by those of the A/WSN $+1 / 244 \mathrm{KO}$ DI virus. As expected from earlier data mice treated with UV-inactivated DI virus were not protected [43, 44]. We have shown previously that animals treated simultaneously with 1/244 DI virus and infectious virus generate protective immunity that prevents disease following subsequent challenge with a high dose of the same virus in the absence of further treatment with DI virus. Animals treated here with 1/244 AUG KO DI virus were solidly immune to further challenge with a high dose of A/WSN showing that they had been infected during the first virus challenge even though they developed no sign of clinical disease (Fig. 2d). These data show that interference in vitro or protection in vivo by $1 / 244$ DI virus does not require expression of a protein product and is a purely RNA-mediated phenomenon.

\section{1/244 DI RNA interferes with packaging of segment 1}

It was reported previously that the interference mediated by a DI virus occured at the level of packaging [57], and we investigated whether this was also the case for DI $1 /$ 244. Human 293T cells were transfected with various amounts of the $1 / 244$ DI plasmid and a constant amount of the plasmids needed for the production of infectious A/ WSN virus. These cells were then co-cultivated with MDCK cells, and at days 1-3 post co-culture the levels of segments 1, 2 and 7 vRNAs in infected cells and in purified virus particles were determined. Previously we showed that influenza vRNAs were only detectable when all of the virus RNA polymerase components were present, demonstrating that the vRNAs are generated by the virus polymerase [46]. 1/244 DI RNA was observed only in cultures transfected with the $1 / 244$ plasmid, confirming that no other segment 1 DI RNA sequences were generated following transfection (Fig. 4a). We determined the effect of 1/244 DI RNA on the packaging efficiency of full-length segment 1 by measuring the ratio of segment 1 to segment 7 in cells and in virions over time in the absence, or presence of increasing levels, of $1 / 244$ DI RNA. Packaging of segment 7 in virions is expected to be unaffected by a segment 1-derived RNA. Therefore, an 


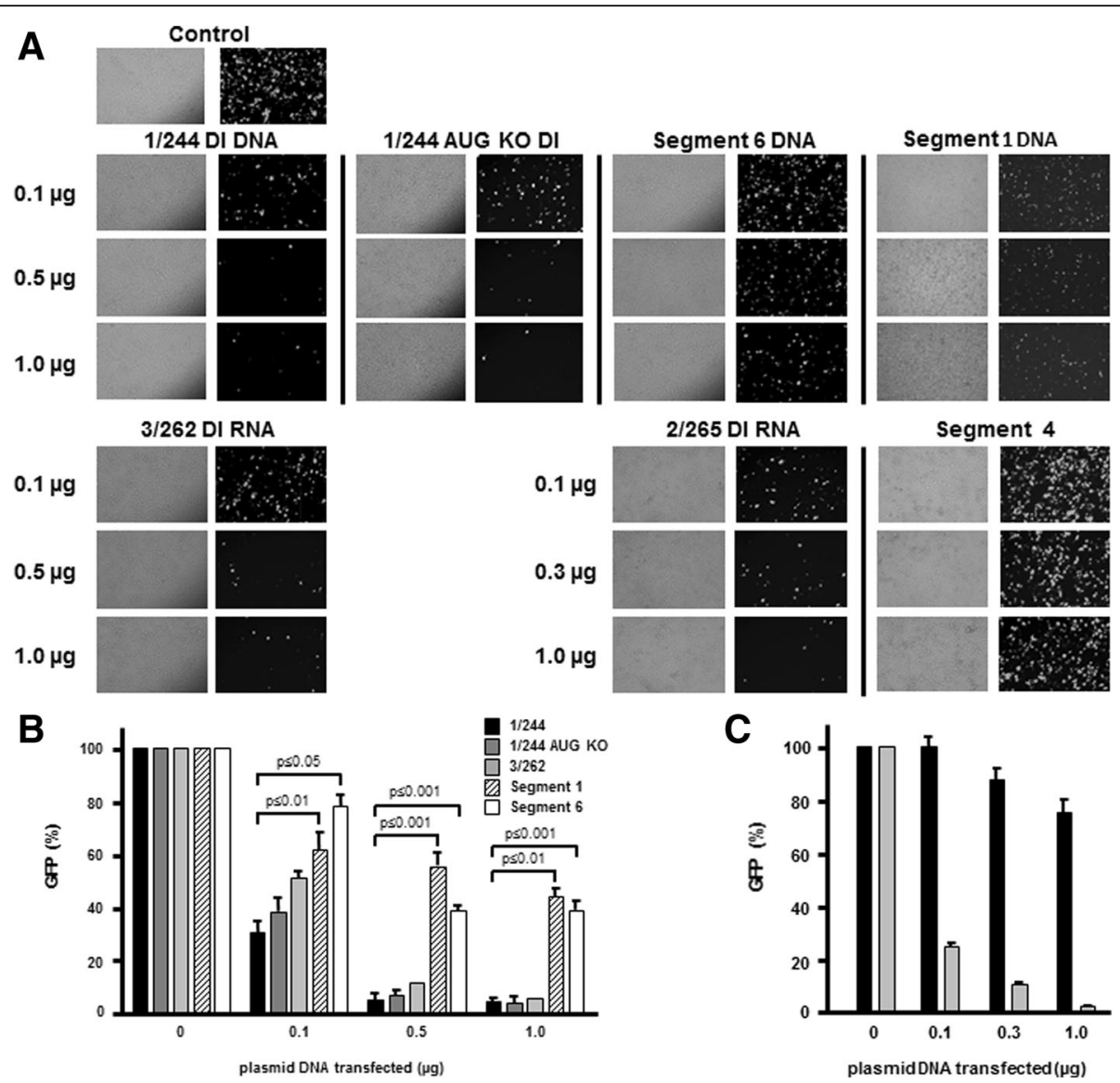

Fig. 3 Effect of increasing amounts of DI and full-length genome segment RNAs on expression of GFP from a segment 1 reporter gene construct. 293 T cells were transfected with the segment 1-GFP plasmid, plasmids expressing PB1, PB2, PA and NP proteins, and increasing amounts of an additional Poll plasmid expressing a DI RNA $(1 / 244,1 / 244$ KO, 2/265 or 3/262) or a full-length vRNA (segment 1, 4 or 6). Cells were examined for fluorescence 2 days after transfection. a. Pairs of cell monolayer images taken by phase-contrast (left) and epifluorescence microscopy (right). The amount of each plasmid expressing the various RNAs used as putative inhibitors is shown on the left. Control cells (top) were transfected with an empty vector $(1 \mu \mathrm{g})$. b. Quantitation of fluorescence in cells generated in the presence of transfected plasmids expressing 1/244 DI, 1/244 KO DI, 3/262 Dl, full-length segment 1 and full-length segment 6 RNAs, as indicated in the key. Columns show the mean of 3 (1/244 RNA, segment 1 and segment 6) or $2(1 / 244$ KO DI RNA and 3/262) independent experiments, and bars are standard errors of the mean. c Quantitation of fluorescence in cells generated in the presence of transfected plasmids expressing full-length segment 4 RNA (black columns) and DI 2/265 RNA (grey columns). Columns show the mean of 2 independent experiments, and bars are standard errors of the mean. Statistical significance determined using a two-tailed Student's $t$ test; $p$ values for specific comparisons are shown

alteration in the ratio of intracellular versus packaged RNA segments indicated whether full-length segment 1 was affected by the presence of the DI RNA. Measuring the ratio of segment 1 to segment 7 also controls for any overall reduction in production of infectious virus particles due to the presence of the DI RNA. As the amount of transfected 1/244 DI plasmid DNA was increased there was a progressive reduction of segment 1 in cells (Fig. 4a upper panel) and a corresponding reduction in virus infectivity on each of the days examined (Fig. 4c). This confirmed that 1/244 DI RNA-mediated interference was taking place. The reduction in infectious particle production was mirrored by a reduction in virion RNA levels, with no segment 1,2 or 7 vRNAs detected in the presence of $1 \mu \mathrm{g}$ of 1/244 DI plasmid DNA (Fig 4a, b, lower panels).
Quantitation showed that in the presence of $1 / 244$ DI RNA, the ratio of full-length segment 1 vRNA: segment 7 vRNA was considerably lower in virions than in cell extracts (Fig. 4d). This established that 1/244 DI RNA acts, at least in part, by selectively excluding full-length cognate segment 1 vRNA from progeny virus particles. The segment 2 vRNA content of virions was not reduced in the presence of increasing amounts of $1 / 244$ plasmid (Fig. 4b, d), confirming that inhibition of packaging of segment 1 by $1 / 244$ DI RNA was specific, and did not extend to other polymerase component-encoding RNA segments. The origin of the band in the total cellular RNA underneath the segment 2 signal is not known, but is likely arise from a non-specific interaction of the probe with cellular RNA. 

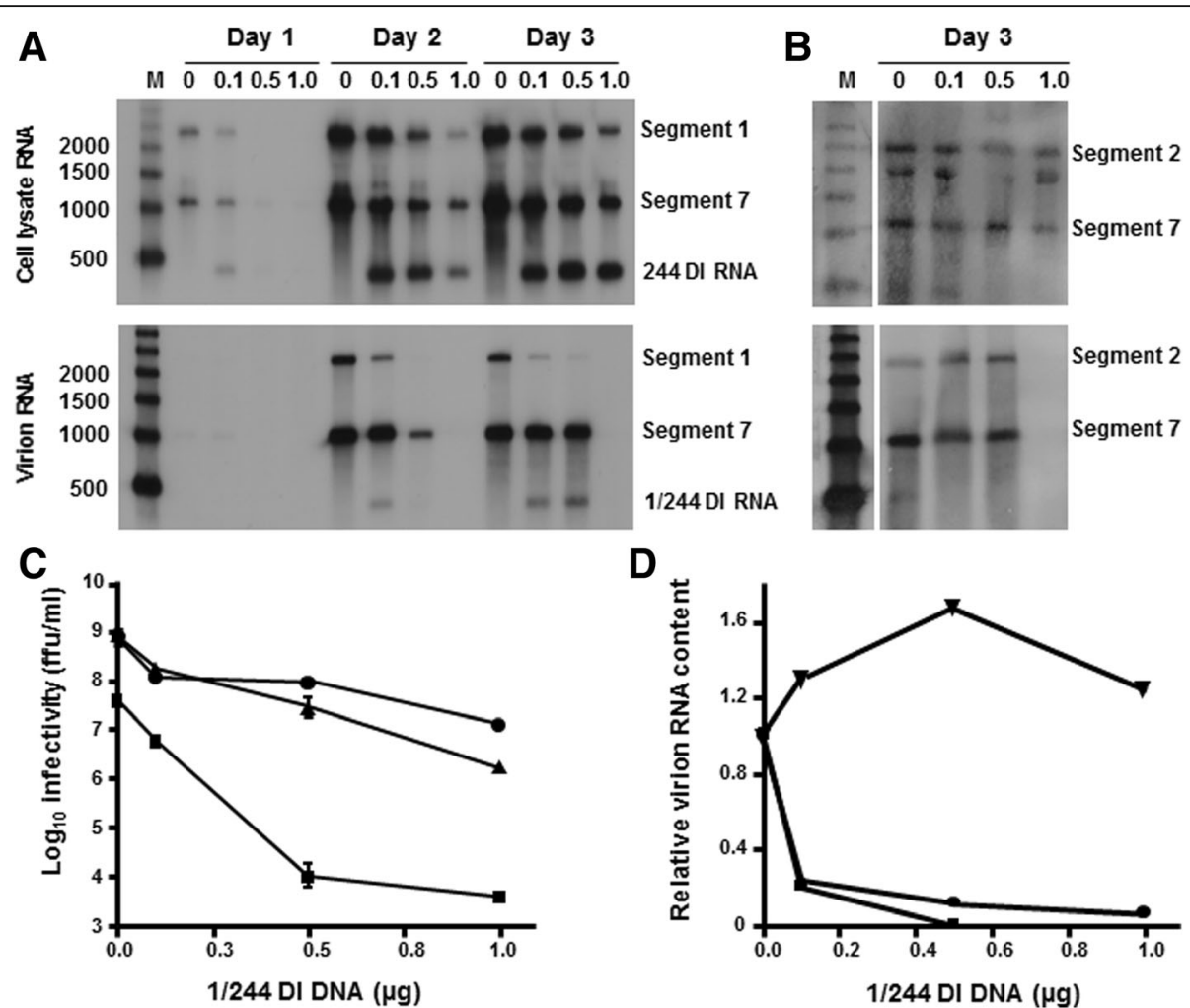

D

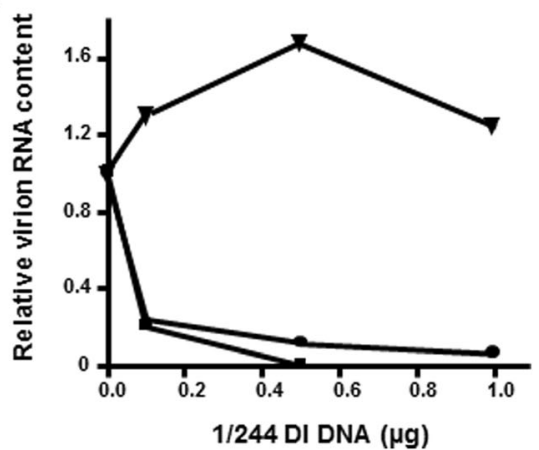

Fig. 4 Effect of DI RNA on the accumulation and encapsidation of viral RNAs. 293T cells were transfected with increasing amounts of the $1 / 244$ DI Poll plasmid $(0,0.1,0.5$ and $1.0 \mu \mathrm{g})$ and a constant amount of the plasmids needed for the expression of infectious A/WSN virus. After cocultivation with MDCK cells RNA was extracted from cells and from virus particles purified from culture fluids. a. RNA extracted from cell lysates (top panel) and virus particles from supernatants (lower panel) at 1,2 and 3 days post co-cultivation was analysed with probes specific for segment 1 RNA, segment 7 RNA, and 1/244 DI RNA. The sizes of RNA markers are shown on the left and the identity of the RNAs on the right. b. Cell lysate RNA and virion RNA extracted on day 3 were analysed with probes specific for segment 2 RNA and segment 7 RNA. c. ANSN infectivity in cell supernatants measured by microplaque assay. The infectivities on $1(\mathbf{\bullet}), 2(\mathbf{\Lambda})$ and $3(\bullet)$ days after co-cultivation are shown. Data are the mean of 2 independent experiments with the bar representing the range. $\mathbf{d}$. The ratios of levels of segment 1 RNA to segment 7 in virions on days $2(\boldsymbol{\bullet})$ and $3(\bullet)$ and of segment 2 RNA to segment 7 in virions on day $3(\boldsymbol{\nabla})$ were determined by densitometry. These values were compared with the equivalent ratios determined from transfected cells. The ratios of segment 1 or segment 2 in virions over total cellular RNA were calculated and normalised to the value determined in the absence of 1/244 DI RNA

\section{Segment 1, 2 or 3 DI RNAs inhibit gene expression from} segment 1

To distinguish between the effects of DI RNA on viral RNA synthesis and RNA packaging, GFP expression from the Seg 1-GFP construct was determined as described above. Human 293T cells were transfected with Seg 1GFP and plasmids expressing PB1, PB2, PA and NP proteins, and GFP fluorescence determined 2 days posttransfection. In addition, increasing amounts of a PolI plasmid expressing either DI $1 / 244$ or an alternative DI RNA (2/265 or 3/262, containing 244, 265 and 262 nt from the 3 ' end of the cognate vRNAs, respectively: Fig 1a) or a fulllength vRNA not expressing a polymerase component (segment 4 or 6) were transfected. In control experiments, increasing amounts of a PolI plasmid encoding full-length vRNA for segment 1 were added to determine whether the presence of segment 1 sequences in general inhibited GFP expression. In the absence of the expression plasmid encoding the PB2 protein no GFP expression was detected confirming the requirement for formation of a functional vRNP complex (data not shown). Figure 3a shows substantial expression of fluorescence in the absence of any DI RNA, while increasing levels of $1 / 244$ DI led to significantly diminished GFP expression. In contrast, inhibition was far less marked when cells were transfected with plasmids that synthesised full-length segments 1,4 or 6 vRNAs. The inhibitory effect of $1 / 244$ DI RNA was highly significantly different to that of segment 6 RNA, with $0.1 \mu \mathrm{g}$ of $1 / 244$ DI plasmid required to inhibit GFP fluorescence by $70 \%$, whereas 10-fold more of the segment 6 plasmid was needed to produce a similar level of inhibition (61-69\%) (Fig. 3b). The presence of $1 \mu \mathrm{g}$ of segment 4 plasmid DNA reduced segment 1-derived gene expression by only $25 \%$ (Fig. 3c). Thus 1/244 DI RNA strongly inhibits gene expression from a segment 1 target RNA. Addition of full-length segment 1 had only a relatively small effect with $1 \mu \mathrm{g}$ plasmid DNA 
reducing GFP fluorescence by $57 \%$, demonstrating that the effect of the DI plasmids was due to their unique sequences and not to a general effect of segment 1 terminal sequences. Further assays showed that the segment 2- and segment 3derived DI RNAs (2/265 and 3/262, respectively) also strongly inhibited GFP fluorescence from the segment 1derived target (Fig. 3a-c), with $1 \mu$ g of the $2 / 265$ and $3 / 262$ DI plasmid DNAs reducing expression to $2 \%$ and $6 \%$ of the control, respectively.

1/244 DI RNA inhibits positive-sense RNA synthesised from genome segments 1, 2 and 3 but from not segment 6

Expression of GFP from the Seg 1-GFP PolI plasmid is dependent on transcription of the negative-sense vRNA into mRNA. However, vRNA is also template for cRNA, which in turn acts as template for the production of more vRNA. Influenza virus mRNA has a 5 '-extension of approximately $12 \mathrm{nt}$ cleaved from host mRNA [10] and is polyadenylatedso the mRNA and cRNA products are distinguishable by size. Using Seg 1-GFP plasmid with the transfection protocol described above, and a primer extension assay that detects vRNA, mRNA and cRNA, we investigated 1/244 DI RNA mediated interference of RNA synthesis (Fig. 5). Viral RNAs were all of the predicted sizes indicating that the templates generated the correct products. RNA levels were normalised to the $5 \mathrm{~S}$ loading control levels before quantitation and the levels of vRNA were also adjusted to account for basal transcription from the target plasmids. Basal transcription was calculated as a percentage of the vRNA:5S RNA ratio in the absence of both the plasmid pcDNA-PB2 (which encodes a critical component of the virus polymerase), and of any interfering RNA (see Fig. 7e, lane 1$)$. This value $(0.93 \%)$ was subtracted from the vRNA levels. Values of zero indicate that no vRNA was detected above the basal level.

Quantitation of the primer extension products showed that mRNA and cRNA levels were considerably more reduced than was vRNA in the presence of $0.1 \mu \mathrm{g}$ to 0.5 $\mu \mathrm{g}$ of $1 / 244$ plasmid DNA (Fig. 5a, b). Addition of $0.1 \mu \mathrm{g}$ of $1 / 244$ plasmid DNA reduced mRNA and cRNA levels to $13 \%$ and $10 \%$ of the control, respectively, while the level of vRNA was reduced to only $54 \%$ of the control (vRNA:mRNA $p=0.0052$, vRNA:cRNA $p=0.016$ ). However, $0.5 \mu \mathrm{g}$ and $1 \mu \mathrm{g}$ of $1 / 244$ reduced the levels of

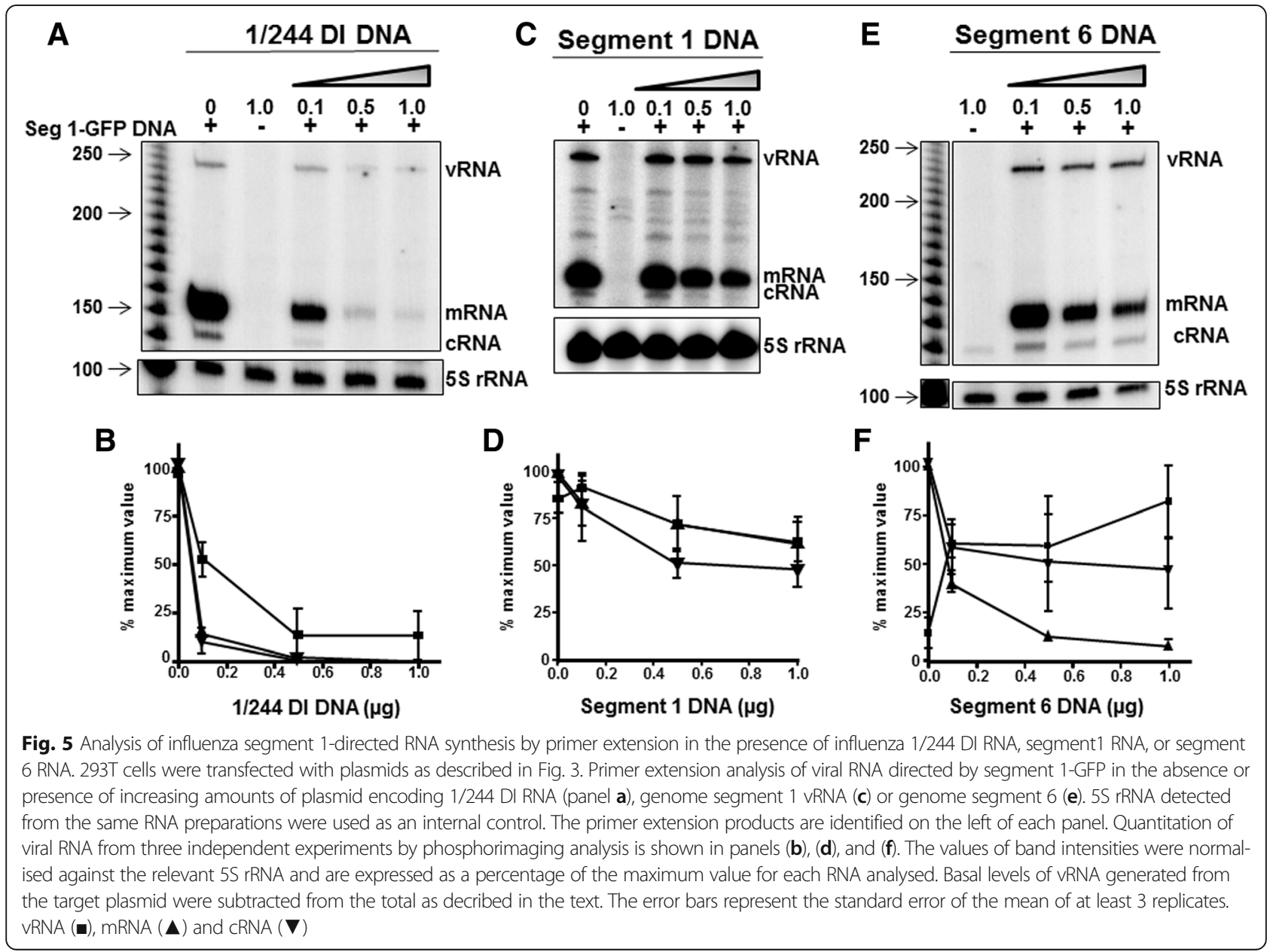


mRNA and cRNA synthesised from segment 1 by $>99 \%$ while levels of vRNA remained at $14 \%$. Thus $1 / 244$ DI RNA has a profound effect on all RNAs synthesised from the segment 1 target, but differentially targets positive-sense RNAs. To control for the specificity of action of the inhibiting RNA, either a segment1 (encoding the intact PB2 gene) or a segment 6 (encoding the NA gene) plasmid was transfected in the place of the DI RNA plasmid. Fig. 5c-f show that segment 1 and 6 RNAs were significantly less effective at inhibiting mRNA, cRNA and vRNA expression from the segment 1 target than was $1 / 244$ DI RNA. This is consistent with the lower level of fluorescence inhibition achieved by the segments 1 and 6 RNA (Fig. 3a). The reduction in the level of segment 1-GFP encoded mRNA and the reduction in GFP fluorescence (Figs. 3 and 5) were strongly positively correlated $\left(\mathrm{R}^{2}=0.90\right.$; data not shown), confirming that fluorescence was a faithful marker of mRNA synthesis.

To determine if $1 / 244$ DI RNA-mediated inhibition of RNA synthesis is segment specific, the segment 1 target (Seg1-GFP) was replaced with a plasmid directing the synthesis of either full-length segment 2, segment 3 or segment 6 vRNAs. Fig. $6 a-d$ shows that 1/244 DI RNA reduced the levels of all three RNAs synthesized by segment 2 or segment 3, whereas segment 6 mRNA production was unaffected even at the highest amount of $1 / 244$ DI plasmid transfected $(1 \mu \mathrm{g})$, cRNA and vRNA levels were reduced to $12 \%$ and $23 \%$ of the control value, respectively. Although inhibition of segment 2-derived RNAs by $1 / 244$ DI RNA (Fig. 6a, b) more closely resembled that seen for Seg1-GFP (Fig. 5a, b) than for segment 3 or 6 target RNAs (Fig. 6c-f), four-fold more 1/244 plasmid DNA was required to reduce segment 2 mRNA levels to $13 \%$ of the control than was needed for Seg 1GFP. The trend of reduction of mRNA, cRNA and vRNA levels with the segment 3 target in duplicate samples was similar though slightly less pronounced (Fig. 7c, d). Overall, the data are consistent with a mechanism whereby $1 / 244$ DI RNA reduces levels of mRNA synthesized from segments 1, 2 and 3, but not from segment 6 .

1/244 DI RNA inhibits synthesis of its own negative-sense vRNA but not its own positive-sense RNA

In light of the ability of $1 / 244$ DI RNA to differentially reduce the level of segment 1-encoded RNAs, we investigated whether the levels of positive- and negative-sense RNAs synthesised from the 1/244 DI RNA itself were also affected. RNA was extracted from $293 \mathrm{~T}$ cells transfected with increasing levels of 1/244 DI RNA, and plasmids expressing PB1, PB2, PA and NP proteins, in the presence or absence of Seg1-GFP DNA (Fig. 7a, c).

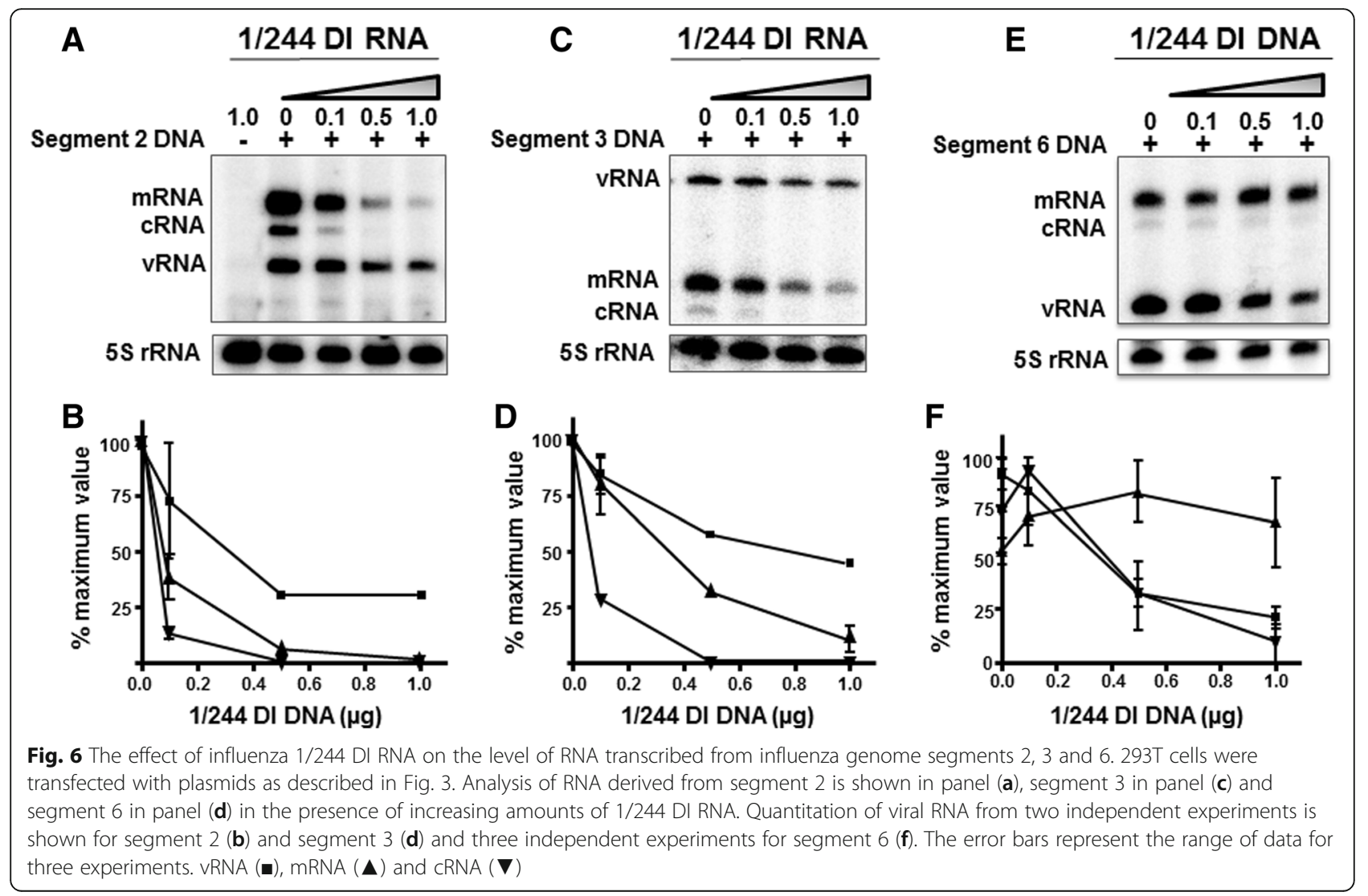




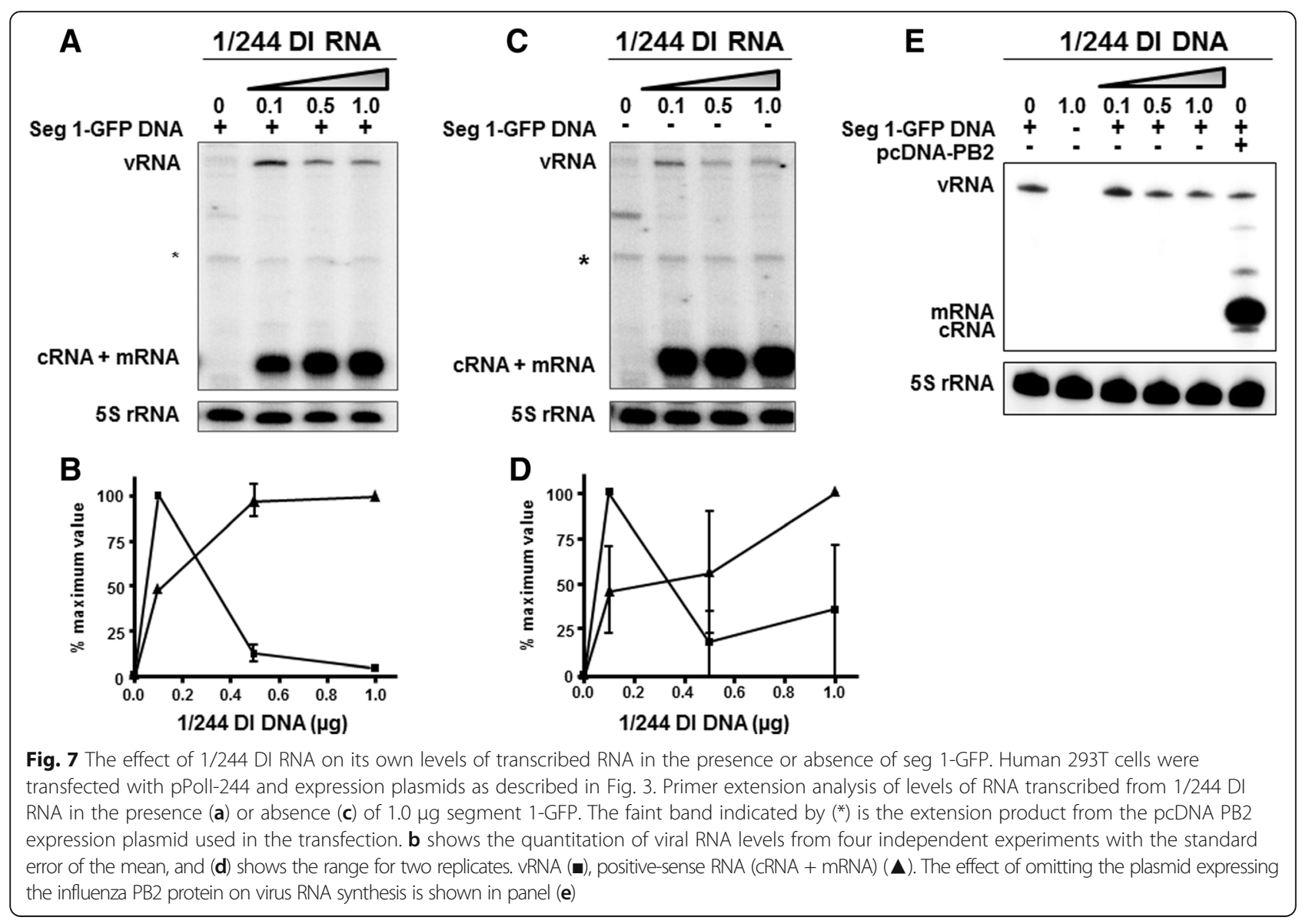

Although we found previously that we could detect vRNA, cRNA and mRNA using primer extension, in these experiments the gel electrophoresis could not distinguish the positive-sense cRNA and mRNA. However, Fig. $7 \mathrm{a}, \mathrm{b}$ show that in the presence of the Seg 1-GFP target, 1/244 DI positive-sense RNA levels increased in proportion to the amount of transfected 1/244 DI plasmid. Thus these were maximal at the same time as the Seg 1-GFP mRNA and cRNA levels were at a minimum as shown in Fig. 5a, b. This shows that $1 / 244$ DI RNA does not inhibit all influenza polymerase-directed transcription. However, the level of $1 / 244$ DI-specific vRNA was reproducibly maximal with $0.1 \mu \mathrm{g} 1 / 244$ plasmid DNA, but decreased to $12 \%$ of the maximum value with $0.5 \mu \mathrm{g}$ plasmid (vRNA: positive-sense RNA $p=0.0002$ ), and to $4 \%$ with $1 \mu \mathrm{g}$ plasmid (Fig. 7b) demonstrating that a high concentration of $1 / 244$ DI RNA reduces the level of its own de novo produced vRNA. When the 1 / 244 plasmid DNA was titrated in cells in the absence of any target RNA the resulting levels of $1 / 244$ positivesense RNA and vRNA were similar to those in the presence of segment 1 target RNA (Fig. 7c, d). The effects of $1 / 244$ DI RNA on RNA synthesis were entirely dependent on transcription of the target RNA carried out by the virus polymerase as omission of the expression plasmid that provides the $\mathrm{PB} 2$ protein that forms part of the virus RNA polymerase, and which is encoded by segment 1, resulted in no expression of Seg1-GFP mRNA or cRNA, although low levels of vRNA transcribed directly from the input plasmid DNA were detected as expected (Fig. 7e).

\section{Discussion}

Despite many years of investigation, the mechanisms of the interfering action of DI influenza viruses in vitro and protection from disease in vivo remain elusive [58-62]. It is commonly held that the small size of the DI RNA allows it to outcompete the full-length genome, and that the proportion of virus particles containing DI genomes simply reflects the relative levels of DI and intact genomes present within infected cells [63]. A second hypothesis is that DI RNA has a comparative advantage over full-length genomes for a limiting viral factor (such as the NP protein) or a host factor [64]. However, there is little experimental evidence to support either of these hypotheses in regard to DI genomes in general, or to influenza DI genomes in particular. A more recent third hypothesis suggests that the influenza DI RNA interferes 
at the level of packaging of genomic RNAs into virions [57]. There have also been suggestions that different influenza DI sequences have different biological properties [41]. Here we investigated the mechanism of action of the cloned influenza A segment 1-derived 1/244 DI virus and demonstrated that its DI RNA inhibits infectious influenza A viruses at the level of packaging of virion RNA and by specific inhibition of virus RNA synthesis.

We have demonstrated that the activity of $1 / 244$ DI is solely an RNA-based phenomenon. DI mRNAs that retain the AUG initiation codon of the major open reading frame have the potential to be translated into truncated peptides, as already demonstrated for some other segment 1-derived DI RNAs [65], and analogous short polypeptides containing the PA protein binding domain of the PB1 protein strongly inhibited influenza A RNA polymerase activity $[66,67]$. A truncated form of the PB2 protein was also shown to be a potent inducer of interferon that could inhibit influenza A replication [68]. Mutation of the AUG initiation codon for PB2 and two further downstream in-frame AUG codons that could direct synthesis of short polypeptides from the PB2 ORF in the 1/244 DI RNA segment (Fig. 1b) generated a 1/ 244 AUG knock-out DI RNA that was replicated and packaged and indistinguishable in action from that of the parental 1/244 DI RNA. Virus containing the $1 / 244$ AUG KO DI RNA protected mice from an influenza challenge to a similar extent to $1 / 244$ DI virus. DI $1 / 244$ KO RNA synthesised vRNA and mRNA to similar levels, and inhibited GFP expression from segment 1 to a similar extent as did 1/244 DI RNA.

Analysis of RNA present in virus particles suggests that $1 / 244$ DI RNA is preferentially packaged over its cognate full-length segment 1 RNA into nascent virions (Fig. 4). Thus, in infected cells $1 / 244$ DI RNA is found as a virion RNP and acts in a segment-specific manner similar to that reported for the segment 1-derived 317 DI RNA in a non-cloned virus population [57], and for cloned 317 DI virus [69]. This is consistent with current models suggesting that influenza genome segments form arrays consisting of a single copy of each segment (whether full-length or DI) and that these arrays act as single structures which become packaged into new virus particles [70-73]. These data indicate that competition for packaging with the cognate full-length genomic RNA is likely to be a common feature of all influenza DI RNAs.

It was not possible to use in vitro infection to study interference by the $1 / 244$ DI genome due to the natural occurrence of DI RNAs in all infectious influenza preparations, their activity masking any signal from the DI RNA synthesized from the transfected plasmid. We therefore used the well-characterised RNP reconstitution assay to investigate viral RNA synthesis in the absence of virion production. While initial transcription events in this system require PolI driven plasmid transcription, for most of the $48 \mathrm{~h}$ time course of the assay virus proteins are synthesised from the newly synthesised virus RNAs as they are in a natural infection. Analysis of the effect of the 1/244 DI genome on mRNA synthesis from a segment 1 target genome RNA, measured directly or by monitoring expression of a reporter gene, showed that 1/244 DI RNA interfered with the synthesis of segment 1 (Fig. 3). The considerably weaker level of inhibition mediated by full-length segments 1,4 and 6 confirmed that this effect was $1 / 244$ DI RNA-specific. Inhibition seen with increasing levels of plasmid DNA expressing genome segments 1,4 and 6 may be due to high levels of these RNAs competing for a limiting factor such as the virus polymerase complex.

The synthesis of positive- (mRNA and cRNA) and negative-sense (vRNA) RNAs are distinct processes $[20,21]$. Data presented here show that $1 / 244$ DI RNA produces positive- and negative-sense RNAs with the predicted sizes and differentially affects the steady state levels of the RNA products expressed by various targets. Increasing amounts of transfected 1/ 244. DI plasmid dramatically reduced full-length segment 1-derived positive-sense mRNA and cRNA levels but four-fold more plasmid DNA was required to reduce negative-sense vRNA to similar levels (Fig. $5 \mathrm{a}, \mathrm{b})$. This contrasts with an earlier report that the segment 1-derived 317 DI virus did not inhibit RNA synthesis, though in that study the DI RNA was not molecularly cloned and has a different sequence to $1 /$ 244 [57]. Surprisingly, 1/244 DI RNA also strongly inhibited mRNA synthesis from segments 2 and 3 (Fig. 6) suggesting that genome segments 1-3 (encoding components of the virus RNA polymerase) share a common feature(s) that permits the inhibitory action of segment 1 DI RNAs. This was reciprocated as DI RNAs 2/265 and 3/262 inhibited GFP expression from the segment 1 target (Fig. 3). This is the first demonstration that an influenza DI RNA can dramatically affect gene expression from a genome segment other than that from which it arose. At levels of $1 /$ 244 DI RNA which strongly reduced the level of target mRNA from segment 1 , its own positive-sense RNA levels were maximal (Fig. 7a, b). Thus, 1/244 DI RNA can transcribe from itself while suppressing synthesis from the target segment 1 RNA.

These data provide the first description of a novel mechanism for preferential production of flu DI RNA compared to RNA production from the cognate genome segment. As the amount of transfected 1/244 DNA was increased there was a proportionate decrease in the mRNA, cRNA and vRNA synthesised by the segment 1 target RNA (Fig. 5) whereas, with transfection of low 
levels of 1/244 DNA all three RNAs transcribed from the 1/244 DI RNA template increased (Fig. 7). However, the situation is complicated as higher levels of $1 / 244$ DI plasmid caused a reduction in the amount of DI vRNA. This reduction is not yet understood. The observed decline in DI vRNA levels suggests there could be an imbalance in the synthesis of the three DI RNAs in which vRNA, which is templated by and therefore dependent on DI cRNA, loses out. This would appear to be a selflimiting phenomenon not previously described for DI virus systems.

The reduction of mRNA levels by $1 / 244$ DI RNA, indicated by the expression of GFP, was not observed when full-length segments 4 or 6 were used as the target, indicating that $1 / 244$ DI RNA does not interfere with all genome segments and appears to act selectively on the synthesis of positive-sense RNA from segments 1, 2 and 3 . Others have shown that segments 1,2 and 3 direct the synthesis of considerably lower levels of mRNA relative to vRNA compared with other genome segments, and it has been suggested that segments 1-3 mRNAs are produced by primary transcription rather than transcription from newly synthesised vRNA [74, 75]. Thus transcription from the three largest genome segments and the other segments appears to differ. Data presented here suggest that DI RNAs derived from segments 1, 2 or 3 suppress transcription from segments 1, 2 and 3 by affecting this transcription process. However, they do not have this effect on segment 6 which, in common with segments $4,5,7$ and 8 , is transcribed primarily from newly synthesised genomes. Large quantities of short RNA molecules, referred to as svRNA or leRNA, are produced during influenza infection [76, 77], and may play a role in the switch from transcription to replication [76]. If correct, this raises the possibility that a DI RNA may serve as the template for the production of svRNAs, which in turn modulate the production of the replication products vRNA, cRNA, and mRNA. The mechanism(s) by which these different synthetic processes in segments 1,2 and 3 are affected is not known, and it will be of interest to investigate if this is common to all influenza DI RNAs or is a property only of certain specific DI RNAs. That is, the huge number of DI RNAs that can be produced during an influenza infection may vary quantitatively and/or qualitatively in the interference they exert.

The ability of influenza DI RNAs to supplant their cognate genome segment during the packaging process explains their amplification in virus preparations. However, data presented here also indicate that the widely held view that interference results solely from the ability of the DI RNA to be replicated faster than the longer, cognate fulllength RNA, while possibly a contributory factor, need not always be the only mechanism involved. Rather, the $1 / 244$
DI RNA specifically targets the synthesis of full-length genome segments 1,2 and 3. Further DI RNAs derived from segments 2 and 3 also inhibit RNA synthesis from full-length segment 1 . Inhibition primarily affects segments $1-3$ as RNA synthesis directed by segment 6 is inhibited to a significantly lesser degree. Taken together, the data suggest that interference depends upon a direct association between each DI RNA and segment 1 . Since all virion and DI RNAs form RNP complexes with NP protein, we suggest that specific interactions reside in the DI RNA itself through an element, which might be a sequence, a motif comprising non-contiguous sequences, or a structural motif. This may be analogous to the association of genome segments through direct RNA: RNA interactions that have been described as a component of virus packaging [72, 78-83].

\section{Conclusions}

The data presented here suggest there may be previously unknown interactions between full-length RNAs 1, 2 or 3 and their cognate DI RNAs, possibly mediated through yet to be identified recognition elements. These interactions appear to confer complex yet highly specific effects on viral transcription, and is therefore likely to be of evolutionary significance to the survival of the virus. This would suggest that DI RNAs are not a mere accident of replication, as frequently proposed, but may have evolved as a non-heritable means of negatively regulating virus infection. These findings take us closer to understanding the evolutionary significance of DI RNAs and their interactions with infectious virus, and nearer to understanding the action of antivirals based on DI RNAs.

\section{Abbreviations \\ CRNA: Positive sense antigenome RNA; Dl: Defective interfering; DIG: Digoxigenin; GFP: Green fluorescent protein; ORF: Open reading frame; PCR: Polymerase chain reaction; RNP: Ribonucleoprotein; VRNA: Negative sense virion genomic RNA; VSV: Vesicular stomatitis virus}

\section{Acknowledgements \\ The authors would like to thank the staff of the University of Warwick Biomedical Support Unit for their assistance.}

\section{Funding}

Medical Research Council (Grant No. G0600832).

\section{Availability of data and materials}

The datasets supporting the conclusions of this article are included within the article.

\section{Authors' contributions}

AJE, NJD, ACM and BM devised the experiments, BM, KB, PS, NJD and AJE carried out experimental work. All authors contributed to writing the manuscript. All authors read and approved the final manuscript.

Ethics approval and consent to participate

Animal work was approved by the University of Warwick Animal Welfare and Ethical Review Board as required by the Animals (Scientific Procedures) Act (1986) governing animal experimentation in the UK. Animals culled for 
humane reasons were subjected to an approved procedure to minimise suffering.

\section{Consent for publication}

All authors have agreed to the publication of this manuscript.

\section{Competing interests}

NJD and AJE have patents for use of DI 1/244 RNA as an antiviral.

\section{Publisher's Note}

Springer Nature remains neutral with regard to jurisdictional claims in published maps and institutional affiliations.

\section{Author details}

${ }^{1}$ Present Address: Department of Medicine, Addenbrooke's Hospital, Hills Road, Cambridge CB2 OQQ, UK. ${ }^{2}$ Present Address: Biomedical Sciences Research Complex, North Haugh, University of St. Andrews, St Andrews KY16 9ST, UK. ${ }^{3}$ Present Address: Public Health England, Porton Down, Salisbury SP4 0JG, UK. ${ }^{4}$ Present Address: Public Health England Birmingham Microbiology, Department of Pathology, Heart of England NHS Foundation Trust, Heartlands Hospital, Bordesley Green East, Salisbury B9 5SS, UK. ${ }^{5}$ School of Life Sciences, University of Warwick, Coventry CV4 7AL, UK.

\section{Received: 22 February 2017 Accepted: 14 July 2017}

\section{Published online: 24 July 2017}

\section{References}

1. Dohner D, Monroe S, Weiss B, Schlesinger S. Oligonucleotide mapping studies of standard and defective Sindbis virus RNA. J Virol. 1979;29:794-8.

2. Kennedy SI. Sequence relationships between the genome and the intracellular RNA species of standard and defective-interfering Semliki Forest virus. J Mol Biol. 1976;108:491-511.

3. Leppert M, Kort L, Kolakofsky D. Further characterization of Sendai virus DI-RNAs: a model for their generation. Cell. 1977;12:539-52.

4. Jennings PA, Finch JT, Winter G, Robertson JS. Does the higher order structure of the influenza virus ribonucleoprotein guide sequence rearrangements in influenza viral RNA? Cell. 1983;34:619-27.

5. Nayak DP, Tobita K, Janda JM, Davis AR, De BK. Homologous interference mediated by defective interfering influenza virus derived from a temperature-sensitive mutant of influenza virus. J Virol. 1978;28:375-86.

6. Dimmock NJ, Easton AJ. Defective interfering influenza virus RNAs: time to reevaluate their clinical potential as broad-spectrum antivirals? J Virol. 2014;88:5217-27.

7. Dimmock NJ, Easton AJ. Cloned defective interfering influenza RNA and a possible pan-specific treatment of respiratory virus diseases. Viruses. 2015;7:3768-88.

8. Noda T, Sagara H, Yen A, Takada A, Kida H, Cheng RH, Kawaoka Y Architecture of ribonucleoprotein complexes in influenza a virus particles. Nature. 2006:439:490-2.

9. Hutchinson EC, von Kirchbach JC, Gog JR, Digard P. Genome packaging in influenza a virus. J Gen Virol. 2010;91:313-28.

10. Palese P, Shaw ML. Orthomyxoviridae: the viruses and their replication. 5th ed: Wolters Kluwer/Lippincott and Williams: Philadelphia; 2007.

11. Huang AS. Defective interfering viruses. Annu Rev Microbiol. 1973;27:101-17.

12. Kolakofsky D. Isolation and characterization of Sendai virus DI-RNAs. Cell. 1976;8:547-55

13. Re GG, Gupta KC, Kingsbury DW. Genomic and copy-back 3' termini in Sendai virus defective interfering RNA species. J Virol. 1983;45:659-64.

14. Nayak DP. Defective interfering influenza viruses. Annu Rev Microbiol. 1980;34:619-44.

15. Boergeling $Y$, Rozhdestvensky TS, Schmolke M, Resa-Infante P, Robeck T, Randau G, Wolff T, Gabriel G, Brosius J, Ludwig S. Evidence for a novel mechanism of influenza virus-induced type I interferon expression by a defective RNA-encoded protein. PLoS Pathog. 2015;11:e1004924.

16. Plotch SJ, Bouloy M, Ulmanen I, Krug RM. A unique cap(m7GpppXm)dependent influenza virion endonuclease cleaves capped RNAs to generate the primers that initiate viral RNA transcription. Cell. 1981;23:847-58.

17. Dias A, Bouvier D, Crépin T, McCarthy AA, Hart DJ, Baudin F, Cusack S, Ruigrok RW. The cap-snatching endonuclease of influenza virus polymerase resides in the PA subunit. Nature. 2009;458:914-8.
18. Fechter P, Mingay L, Sharps J, Chambers A, Fodor E, Brownlee GG. Two aromatic residues in the PB2 subunit of influenza a RNA polymerase are crucial for cap binding. J Biol Chem. 2003;278:20381-8.

19. Guilligay D, Tarendeau F, Resa-Infante P, Coloma R, Crepin T, Sehr P, Lewis J, Ruigrok RW, Ortin J, Hart DJ, Cusack S. The structural basis for cap binding by influenza virus polymerase subunit PB2. Nat Struct Mol Biol. 2008;15:500-6.

20. Jorba N, Coloma R, Ortin J. Genetic trans-complementation establishes a new model for influenza virus RNA transcription and replication. PLoS Pathog. 2009:5:e1000462

21. Yuan P, Bartlam M, Lou Z, Chen S, Zhou J, He X, Lv Z, Ge R, Li X, Deng T, et al. Crystal structure of an avian influenza polymerase $P A(N)$ reveals an endonuclease active site. Nature. 2009;458:909-13.

22. Vreede FT, Brownlee GG. Influenza virion-derived viral ribonucleoproteins synthesize both mRNA and cRNA in vitro. J Virol. 2007;81:2196-204.

23. Resa-Infante $P$, Jorba N, Coloma R, Ortin J. The influenza virus RNA synthesis machine: advances in its structure and function. RNA Biol. 2011:8:207-15.

24. Lee MK, Bae SH, Park CJ, Cheong HK, Cheong C, Choi BS. A single-nucleotide natural variation (U4 to $C 4)$ in an influenza a virus promoter exhibits a large structural change: implications for differential viral RNA synthesis by RNAdependent RNA polymerase. Nucleic Acids Res. 2003;31:1216-23.

25. Fodor E, Pritlove DC, Brownlee GG. The influenza virus panhandle is involved in the initiation of transcription. J Virol. 1994;68:4092-6.

26. Tiley LS, Hagen M, Matthews JT, Krystal M. Sequence-specific binding of the influenza virus RNA polymerase to sequences located at the $5^{\prime}$ ends of the viral RNAs. J Virol. 1994;68:5108-16.

27. González S, Ortín J. Characterization of influenza virus PB1 protein binding to viral RNA: two separate regions of the protein contribute to the interaction domain. J Virol. 1999;73:631-7.

28. González S, Ortín J. Distinct regions of influenza virus PB1 polymerase subunit recognize vRNA and cRNA templates. EMBO J. 1999;18:3767-75.

29. Mena I, Jambrina E, Albo C, Perales B, Ortín J, Arrese M, Vallejo D, Portela A. Mutational analysis of influenza a virus nucleoprotein: identification of mutations that affect RNA replication. J Virol. 1999;73:1186-94.

30. Falcón AM, Marión RM, Zürcher T, Gómez P, Portela A, Nieto A, Ortín J. Defective RNA replication and late gene expression in temperature-sensitive influenza viruses expressing deleted forms of the NS1 protein. J Virol. 2004; 78:3880-8.

31. Thierry F, Danos $O$. Use of specific single stranded DNA probes cloned in M13 to study the RNA synthesis of four temperature-sensitive mutants of HK/68 influenza virus. Nucleic Acids Res. 1982;10:2925-38.

32. Robb NC, Smith M, Vreede FT, Fodor E. NS2/NEP protein regulates transcription and replication of the influenza virus RNA genome. J Gen Virol. 2009;90:1398-407

33. Rao DD, Huang AS. Interference among defective interfering particles of vesicular stomatitis virus. J Virol. 1982;41:210-21.

34. Schubert M, Lazzarini RA. Structure and origin of a snapback defective interfering particle RNA of vesicular stomatitis virus. J Virol. 1981;37:661-72.

35. Pringle CR. Rhabdovirus genetics. New York: Plenum Press; 1987.

36. Whelan SPJ, Wertz GW. Defective interfering particles of vesicular stomatitis virus: functions of the genomic termini. Semin Virol. 1997:8:131-9.

37. Finke S, Conzelmann KK. Virus promoters determine interference by defective RNAs: selective amplification of mini-RNA vectors and rescue from cDNA by a 3' copy-back ambisense rabies virus. J Virol. 1999;73:3818-25.

38. Amesse LS, Pridgen CL, Kingsbury DW. Sendai virus DI RNA species with conserved virus genome termini and extensive internal deletions. Virology. 1982;118:17-27

39. Leppert M, Kolakofsky D. Effect of defective interfering particles on plusand minus- strand leader RNAs in vesicular stomatitis virus-infected cells. J Virol. 1980;35:704-9

40. Giachetti C, Holland JJ. Vesicular stomatitis virus and its defective interfering particles exhibit in vitro transcriptional and replicative competition for purified L-NS polymerase molecules. Virology. 1989;170:264-7.

41. Duhaut SD, Dimmock NJ. Heterologous protection of mice from a lethal human H1N1 influenza a virus infection by H3N8 equine defective interfering virus: comparison of defective RNA sequences isolated from the DI inoculum and mouse lung. Virology. 1998;248:241-53.

42. Dimmock NJ, Dove BK, Meng B, Scott PD, Taylor I, Cheung L, Hallis B, Marriott AC, Carroll MW, Easton AJ. Comparison of the protection of ferrets against pandemic 2009 influenza a virus (H1N1) by 244 DI influenza virus and oseltamivir. Antivir Res. 2012;96:376-85 
43. Easton AJ, Scott PD, Edworthy NL, Meng B, Marriott AC, Dimmock NJ. A novel broad-spectrum treatment for respiratory virus infections: influenza-based defective interfering virus provides protection against pneumovirus infection in vivo. Vaccine. 2011;29:2777-84.

44. Scott PD, Meng B, Marriott AC, Easton AJ, Dimmock NJ. Defective interfering influenza a virus protects in vivo against disease caused by a heterologous influenza B virus. J Gen Virol. 2011;92:2122-32.

45. Neumann G, Watanabe T, Ito H, Watanabe S, Goto H, Gao P, Hughes M, Perez DR Donis R, Hoffmann E, et al. Generation of influenza a viruses entirely from cloned cDNAs. Proc Natl Acad Sci U S A. 1999;96:9345-50.

46. Duhaut SD, Dimmock NJ. Defective segment 1 RNAs that interfere with production of infectious influenza a virus require at least 150 nucleotides of $5^{\prime}$ sequence: evidence from a plasmid-driven system. J Gen Virol. 2002;83:403-11.

47. Duhaut S, Dimmock NJ. Approximately 150 nucleotides from the $5^{\prime}$ end of an influenza a segment 1 defective virion RNA are needed for genome stability during passage of defective virus in infected cells. Virology. 2000;275:278-85.

48. Mann A, Marriott AC, Balasingam S, Lambkin R, Oxford JS, Dimmock NJ. Interfering vaccine (defective interfering influenza a virus) protects ferrets from influenza, and allows them to develop solid immunity to reinfection. Vaccine. 2006;24:4290-6.

49. Subbarao K, Chen H, Swayne D, Mingay L, Fodor E, Brownlee G, Xu X, Lu X, Katz J, Cox N, Matsuoka Y. Evaluation of a genetically modified reassortant H5N1 influenza a virus vaccine candidate generated by plasmid-based reverse genetics. Virology. 2003;305:192-200.

50. Rehwinkel J, Tan CP, Goubau D, Schulz O, Pichlmair A, Bier K, Robb N, Vreede F, Barclay W, Fodor E, Reis E Sousa C. RIG-I detects viral genomic RNA during negative-strand RNA virus infection. Cell. 2010;140:397-408.

51. Duhaut SD, Dimmock NJ. Defective influenza a virus generated entirely from plasmids: its RNA is expressed in infected mouse lung and modulates disease. J Virol Methods. 2003;108:75-82.

52. Carr SM, Carnero E, García-Sastre A, Brownlee GG, Fodor E. Characterization of a mitochondrial-targeting signal in the PB2 protein of influenza viruses. Virology. 2006;344:492-508.

53. Long JS, Giotis ES, Moncorgé $O$, Frise R, Mistry B, James J, Morisson M, lqbal M, Vignal A, Skinner MA, Barclay WS. Species difference in ANP32A underlies influenza a virus polymerase host restriction. Nature. 2016;529:101-4.

54. Te Velthuis AJ, Robb NC, Kapanidis AN, Fodor E. The role of the priming loop in influenza a virus RNA synthesis. Nat Microbiol. 2016;1:16029.

55. Czudai-Matwich V, Otte A, Matrosovich M, Gabriel G, Klenk HD. PB2 mutations D701N and S714R promote adaptation of an influenza H5N1 virus to a mammalian host. J Virol. 2014;88:8735-42.

56. Turrell $L$, Hutchinson EC, Vreede FT, Fodor E. Regulation of influenza a virus nucleoprotein oligomerization by phosphorylation. J Virol. 2015;89:1452-5.

57. Duhaut SD, McCauley JW. Defective RNAs inhibit the assembly of influenza virus genome segments in a segment-specific manner. Virology. 1996;216:326-37.

58. von Magnus P. Studies on interference in experimental influenza. I. Biological observations. Arkiv fur Kemi, Mineralogi och Geologi. 1947;24b:1-6.

59. von Magnus P. Incomplete forms of influenza virus. Adv Virus Res. 1954;21:59-79.

60. Bangham CRM, Kirkwood TBL. Defective interfering particles and virus evolution. Trends Microbiol. 1993;1:260-4.

61. Holland JJ. Generation and replication of defective viral genomes. In: Fields BN Knipe DM, editors. Virology. 2nd ed. New York: Raven Press; 1990. p. 77-99.

62. Dimmock NJ. Antiviral activity of defective interfering influenza virus in vivo. In: Myint S, Taylor-Robinson D, editors. Viral and other infections of the respiratory tract. London: Chapman and Hall; 1996. p. 421-45.

63. Roux L, Simon AE, Holland JJ. Effects of defective interfering viruses on viral replication and pathogenesis in vitro and in vivo. Adv Virus Res. 1991;40:181-211.

64. Laske T, Heldt FS, Hoffmann H, Frensing T, Reichl U. Modeling the intracellular replication of influenza a virus in the presence of defective interfering RNAs. Virus Res. 2015;213:90-9.

65. Akkina RK, Chambers TM, Nayak DP. Expression of defective-interfering influenza virus specific transcripts and polypeptides in infected cells. J Virol. 1984;51:395-403.

66. Mänz B, Götz V, Wunderlich K, Eisel J, Kirchmair J, Stech J, Stech O, Chase G, Frank R, Schwemmle M. Disruption of the viral polymerase complex assembly as a novel approach to attenuate influenza a virus. J Biol Chem. 2011;286:8414-24.

67. Wunderlich K, Mayer D, Ranadheera C, Holler A-S, Mänz B, Martin A, Chase G, Tegge W, Frank R, Kessler U, Schwemmle M. Identification of a PA-binding peptide with inhibitory activity against influenza $a$ and $B$ virus replication. PLoS One. 2009;4:e7517.
68. Boergeling Y, Rozhdestvensky TS, Schmolke M, Resa-Infante P, Robeck T, Randau G, Wolff T, Gabriel G, Brosius J, Ludwig S. Evidence for a novel mechanism of influenza virus-induced type I interferon expression by a defective RNA-encoded protein. PLoS Pathog. 2015;11(5):e1004924.

69. Duhaut SD, Dimmock NJ. Defective segment 1 RNAs that interfere with the production of infectious influenza virus require at least 150 nucleotides of $5^{\prime}$ sequence: evidence from a plasmid-driven system. J Gen Virol. 2002;83:403-11.

70. Noda T, Sagara H, Yen A, Takada A, Kida H, Cheng RH, Kawaoka Y. Architecture of ribonucleoprotein complexes in influenza a virus particles. Nature (London). 2006:439:490-2.

71. Harris A, Cardone G, Winkler DC, Heymann JB, Brecher M, White JM, Steven AC Influenza virus pleiomorphy characterized by cryoelectron tomography. Proc Natl Acad Sci U S A. 2006;103:19123-7.

72. Chou $Y Y$, Heaton NS, Gao Q, Palese P, Singer RH, Singer R, Lionnet T. Colocalization of different influenza viral RNA segments in the cytoplasm before viral budding as shown by single-molecule sensitivity FISH analysis. PLoS Pathog. 2013;9:e1003358.

73. Nakatsu S, Sagara H, Sakai-Tagawa Y, Sugaya N, Noda T, Kawaoka Y. Complete and incomplete genome packaging of influenza a and B viruses. MBio. 2016;7:e01248-16.7

74. Smith GL, Hay AJ. Replication of the influenza virus genome. Virology. 1982:118:96-108.

75. Hatada E, Hasegawa M, Mukaigawa J, Shimizu K, Fukuda R. Control of influenza virus gene expresssion: quantitative analysis of each viral RNA species in infected cells. J Biochem. 1989;105:537-46.

76. Perez JT, Varble A, Sachidanandam R, Zlatev I, Manoharan M, García-Sastre A, tenOever BR. Influenza a virus-generated small RNAs regulate the switch from transcription to replication. Proc Natl Acad Sci U S A. 2010;107:11525-30.

77. Umbach JL, Yen H-L, Poon LLM, Cullen BR. Influenza a virus expresses high levels of an unusual class of small viral leader RNAs in infected cells. MBio. 2010;1:e00204-10.

78. Cobbin JC, Ong C, Verity E, Gilbertson BP, Rockman SP, Brown LE. Influenza virus PB1 and neuraminidase gene segments can cosegregate during vaccine reassortment driven by interactions in the PB1 coding region. J Virol. 2014:88:8971-80.

79. Essere B, Yver M, Gavazzi C, Terrier O, Isel C, Fournier E, Giroux F, Textoris J, Julien T, Socratous $C$, et al. Critical role of segment-specific packaging signals in genetic reassortment of influenza a viruses. Proc Natl Acad Sci U S A. 2013;110:E3840-8.

80. Fournier E, Moules V, Essere B, Paillart JC, Sirbat JD, Cavalier A, Rolland JP, Thomas D, Lina B, Isel C, Marquet R. Interaction network linking the human H3N2 influenza a virus genomic RNA segments. Vaccine. 2012;30:7359-67.

81. Gavazzi C, Yver M, Isel C, Smyth RP, Rosa-Calatrava M, Lina B, Moulès V, Marquet R. A functional sequence-specific interaction between influenza a virus genomic RNA segments. Proc Natl Acad Sci U S A. 2013;110:16604-9.

82. Gavazzi C, Isel C, Fournier E, Moules V, Cavalier A, Thomas D, Lina B, Marquet R. An in vitro network of intermolecular interactions between viral RNA segments of an avian H5N2 influenza a virus: comparison with a human H3N2 virus. Nucleic Acids Res. 2013;41:1241-54.

83. Gerber M, Isel C, Moules $V$, Marquet R. Selective packaging of the influenza a genome and consequences for genetic reassortment. Trends Microbiol. 2014:22:446-55.

\section{Submit your next manuscript to BioMed Central and we will help you at every step:}

- We accept pre-submission inquiries

- Our selector tool helps you to find the most relevant journal

- We provide round the clock customer support

- Convenient online submission

- Thorough peer review

- Inclusion in PubMed and all major indexing services

- Maximum visibility for your research

Submit your manuscript at www.biomedcentral.com/submit 